\title{
Triggered alternans in an ionic model of ischemic cardiac ventricular muscle
}

\author{
Humberto Arce ${ }^{\text {a) }}$ and Alejandro López \\ Departamento de Física, Facultad de Ciencias, Universidad Nacional Autónoma de México, \\ Apartado Postal 70-542, 04510 México, Distrito Federal, México \\ Michael R. Guevara \\ Department of Physiology and Centre for Nonlinear Dynamics in Physiology and Medicine, \\ McGill University, 3655 Sir William Osler Promenade, Montréal, Québec H3G 1Y6, Canada
}

(Received 4 March 2002; accepted 19 June 2002; published 23 August 2002)

\begin{abstract}
It has been known for several decades that electrical alternans occurs during myocardial ischemia in both clinical and experimental work. There are a few reports showing that this alternans can be triggered into existence by a premature ventricular contraction. Detriggering of alternans by a premature ventricular contraction, as well as pause-induced triggering and detriggering, have also been reported. We conduct a search for triggered alternans in an ionic model of ischemic ventricular muscle in which alternans has been described recently: a one-dimensional cable of length $3 \mathrm{~cm}$, containing a central ischemic zone $1 \mathrm{~cm}$ long, with $1 \mathrm{~cm}$ segments of normal (i.e., nonischemic) tissue at each end. We use a modified form of the Luo-Rudy [Circ. Res. 68, 1501-1526 (1991)] ionic model to represent the ventricular tissue, modeling the effect of ischemia by raising the external potassium ion concentration $\left(\left[\mathrm{K}^{+}\right]_{o}\right)$ in the central ischemic zone. As $\left[\mathrm{K}^{+}\right]_{o}$ is increased at a fixed pacing cycle length of $400 \mathrm{~ms}$, there is first a transition from 1:1 rhythm to alternans or $2: 2$ rhythm, and then a transition from $2: 2$ rhythm to $2: 1$ block. There is a range of $\left[\mathrm{K}^{+}\right]_{o}$ over which there is coexistence of 1:1 and 2:2 rhythms, so that dropping a stimulus from the periodic drive train during 1:1 rhythm can result in the conversion of 1:1 to $2: 2$ rhythm. Within the bistable range, the reverse transition from 2:2 to 1:1 rhythm can be produced by injection of a well-timed extrastimulus. Using a stimulation protocol involving delivery of pre- and post-mature stimuli, we derive a one-dimensional map that captures the salient features of the results of the cable simulations, i.e., the $\{1: 1 \rightarrow 2: 2 \rightarrow 2: 1\}$ transitions with $\{1: 1 \leftrightarrow 2: 2\}$ bistability. This map uses a new index of the global activity in the cable, the normalized voltage integral. Finally, we put forth a simple piecewise linear map that replicates the $\{1: 1 \leftrightarrow 2: 2\}$ bistability observed in the cable simulations and in the normalized voltage integral map. (c) 2002 American Institute of Physics. [DOI: $10.1063 / 1.1499275]$
\end{abstract}

Heart attack is a leading cause of death. Death during a heart attack is often due to a disturbance in the rhythm of the heartbeat ("cardiac arrhythmia"). Arrhythmias arise in this context because of the interruption in the flow of blood to the heart muscle ("myocardial ischemia"). It has been known for several decades that a beat-to-beat alternation in the pulse or the electrocardiogram ("alternans") can be seen at the onset of ischemia, just before the arrhythmias first start to appear. While there has been much speculation about a cause-and-effect relationship between the alternans and the arrhythmias, there is as yet no firm evidence for this hypothesis. There is also some evidence that alternans can start up during ischemia immediately following the occurrence of a premature ventricular beat ("triggered alternans"). Again, the exact nature of any causal connection between the premature beat and the triggering of the alternans is un-

a) Address for reprints: H. Arce, Departamento de Física, Facultad de Ciencias, Universidad Nacional Autónoma de México, Apartado Postal 70-542, 04510 México, Distrito Federal, México. Electronic mail: jhar@hp.fciencias.unam.mx certain. We therefore undertook to see whether we could shed some light on triggered alternans by trying to elicit it in an ionic model of ischemic ventricular muscle.

\section{INTRODUCTION}

Electrical alternans is a cardiac arrhythmia in which there is a beat-to-beat alternation in the shape of one or more of the electrocardiographic complexes. Alternans rhythms in which there is frank alternation of some component of the electrocardiogram or electrogram (e.g., ST-segment, T-wave, ventricular gradient) commonly occur during acute myocardial ischemia in both clinical (see Refs. 2-13 in Ref. 1) and experimental ${ }^{2-9}$ work. At times, the alternans can be so small in magnitude that it is not visible to the naked eye, and signal processing techniques (e.g., power spectrum, ${ }^{10-12}$ complex demodulation, ${ }^{13}$ or Karhunen-Loèvre decomposition ${ }^{14}$ ) must be used to establish its existence. There has been much recent interest in detecting this occult alternans to identify patients at risk of arrhythmia in ischemia as well as in other situations. ${ }^{10-16}$ 
During experimental work on acute coronary ischemia, the appearance of an alternans rhythm can sometimes be immediately preceded by a spontaneous premature ventricular contraction (PVC). ${ }^{2,4,8,9}$ One might reasonably think that this finding is simply a coincidence-i.e., that the PVC just happened to come along just by chance at a time when alternans would have started up spontaneously anyway. However, the onset of alternans immediately following a PVC was seen tens of times in one study, ${ }^{8}$ in 21 of 31 experiments in another study, ${ }^{4}$ and in 4 out of 8 animals in yet another study. ${ }^{2}$ There is thus certainly a causal relationship between the PVC and the alternans: hence the term "triggered alternans," 8 which we adopt. Alternans can also cease immediately following a spontaneous $\mathrm{PVC}^{2,4}$ following an externally induced extrasystole, ${ }^{8}$ or following a deliberate pause in stimulation. ${ }^{8}$ We refer to this phenomenon as "detriggering."

It has been known for some time that alternans can exist in situations where inhomogeneity does not play a role in its induction: e.g., single ventricular cells, ${ }^{17-21}$ ionic models of space-clamped ventricular membrane, ${ }^{17,20,22-26}$ ionic models of homogeneous one-dimensional strands ${ }^{27-30}$ and 2-dimensional sheets ${ }^{29,31}$ of ventricular tissue, a coupled-map lattice, ${ }^{30}$ and simple one-dimensional finite-difference equations stemming from experimental ${ }^{32-35}$ and modeling ${ }^{22,27}$ work. Alternans has only more recently been seen in models of inhomogeneous ventricular muscle. ${ }^{1,29,31,36}$ We thus decided to investigate whether triggered alternans could also be seen in a model of ischemic ventricular muscle.

\section{METHODS}

We study an ionic model of a one-dimensional strand of normal ventricular myocardium, with an area of elevated $\left[\mathrm{K}^{+}\right]_{o}$ embedded within its interior to represent the ischemic zone. ${ }^{1,37-43}$ We use a "first-generation" model of the ventricular membrane ionic currents, that due to Luo and Rudy, ${ }^{44}$ avoiding use of one of the more recent "secondgeneration" models.

In their original formulation, second-generation models of space-clamped cardiac membrane suffer from two deficiencies: (i) degeneracy, with nonuniqueness of equilibria (steady-states and limit cycles), ${ }^{45-48}$ and (ii) very slow longterm drifts in the variables. ${ }^{41,47,49-52}$ The degeneracy can be removed by reformulating these models as differentialalgebraic systems, ${ }^{45-48,52}$ rather than as fully-differential systems, which is the way they were originally formulated. In one model of spontaneous activity of the sinus node, in which external stimuli were not delivered, the strategy of employing the differential-algebraic formulation also abolished drift. ${ }^{47}$ Drift in another unstimulated sinus node model was removed by exquisitely adjusting two parameters, maintaining the original fully-differential formulation. ${ }^{49}$ In a model of quiescent ventricular muscle, it has been shown that the drift is abolished in both the fully-differential and the algebraic-differential formulations, provided that one takes into account the ion species injected by the constant-current stimulus pulses (see Fig. 5 of Ref. 52). However, it is not clear from this paper whether drift was originally present in the differential-algebraic case before stimulus-current tracking was incorporated. This procedure involving keeping track of the stimulus current is obviously incapable of removing the drifts seen in the fully-differential formulation of spontaneously active models, ${ }^{49-51}$ where no stimuli are injected. If the fully-differential formulation is used, one is still left with a system that is degenerate, even if one keeps track of the stimulus current (see, e.g., Fig. 6A of Ref. 52). Finally, as previously noted ${ }^{52}$ should one adopt stimulus-current tracking for a cable simulation using the fully-differential formulation, or even using the differential-algebraic formalism (if stimulus-current tracking is indeed needed to remove drift in the space-clamped case), a term to account for intercellular diffusion of the injected ion species and for other ionic species might then have to be added to avoid drift. Given all the above uncertainties and complications, we decided to use a first-generation model. We select the LuoRudy (LR) model because it has $\left[\mathrm{K}^{+}\right]_{o}$ as a parameter, which is essential for our modeling of the ischemic zone.

One deficiency of the space-clamped LR model, which is carried over from the Beeler-Reuter model ${ }^{53}$ from which it is derived, is that the time-constants for the activation and inactivation of the slow inward $\mathrm{Ca}^{++}$current $\left(I_{s}\right)$ are an order of magnitude too large. We have thus decreased the time constant for the activation of $I_{s}\left(\tau_{d}\right)$ by a factor of 10 , which then puts $\tau_{d}$ into the physiologic range. ${ }^{41,54}$ As in our prior work on modeling alternans in ischemic muscle, ${ }^{1}$ we leave the inactivation time-constant of $I_{s}\left(\tau_{f}\right)$ unchanged, since reducing it results in the level of the plateau of the action potential being unrealistically depressed. To describe the other currents we have used the equations appearing in Table I and the body of the text of Luo and Rudy (1991). ${ }^{44}$ It has been noted previously ${ }^{41}$ that using the equations in Table I results in current-voltage relationships for $I_{\mathrm{K} 1}$ and $I_{\mathrm{K} 1(T)}$ that are different from those shown in Figs. 2 and $3 \mathrm{~B}$ of Luo and Rudy (1991), respectively. The ionic concentrations given in Ref. 44 are used to calculate the reversal potentials $E_{\mathrm{Na}}, E_{\mathrm{K}}$, and $E_{\mathrm{K} 1}$ (we take $R T / F=26.7 \mathrm{mV}$ for these calculations). In the steady state, the action potential duration (measured between the upstroke and the crossing through of $-60 \mathrm{mV}$ on the repolarizing limb of the action potential) is reduced to $\sim 237 \mathrm{~ms}$ from $\sim 290 \mathrm{~ms}$ in the standard LR space-clamped model when paced at a basic cycle length of $400 \mathrm{~ms}$.

We model a one-dimensional strand of ventricular muscle by the one-dimensional cable equation, ${ }^{55}$

$$
\frac{\partial^{2} V}{\partial x^{2}}=\rho S_{\nu}\left(C_{m} \frac{\partial V}{\partial t}+I_{\text {ion }}\right),
$$

where $V$ is the transmembrane potential $(\mathrm{mV}), x$ is the spatial coordinate in the strand $(\mathrm{cm}), \rho$ is the effective longitudinal resistivity $(0.2 \mathrm{k} \Omega \mathrm{cm}), S_{\nu}$ is the surface-to-volume ratio $\left(5000 \mathrm{~cm}^{-1}\right), C_{m}$ is the specific membrane capacitance $\left(1 \mu \mathrm{F} \mathrm{cm}^{-2}\right), t$ is time (ms), and $I_{\text {ion }}$ is the total ionic current $\left(\mu \mathrm{A} \mathrm{cm}^{-2}\right)$ given by our modified LR model. We use an explicit integration scheme, with forward Euler integration for the internal calcium concentration, and an exact analytic 
formula for the activation and inactivation variables (for further details of the integration scheme, see Refs. 1, 41). The temporal integration step-size $(\Delta t)$ is $0.01 \mathrm{~ms}$ and the spatial integration step-size $(\Delta x)$ is $0.01 \mathrm{~cm}$. A lookup table with linear interpolation (voltage-step $=0.2 \mathrm{mV}$ ) was used to calculate the asymptotic values and the time constants of the activation and inactivation variables. L'Hôpital's rule was used where needed to evaluate indeterminate forms.

The one-dimensional space-constant is $\lambda=\left(R_{m} / \rho S_{\nu}\right)^{1 / 2}$ $\cong 0.06 \mathrm{~cm}$, where $R_{m}$ is the specific membrane resistance $\left(3.55 \mathrm{k} \Omega \mathrm{cm}^{2}\right.$ at the nominal LR value of $\left[\mathrm{K}^{+}\right]_{o}$ of $\left.5.4 \mathrm{mM}\right)$. The discretization factor $(\Delta x / \lambda)$ is thus $\sim 0.17$, at which point the numerical error is acceptable, as we show below (Fig. 1). The diffusion constant $D=1 /\left(\rho S_{\nu} C\right)$ $=10^{-3} \mathrm{~cm}^{2} \mathrm{~ms}^{-1}$, so that the von Neumann linear stability criterion $\left[(\Delta x)^{2} / \Delta t>4 D\right]$ is satisfied. Sealed-end (i.e., Dirichlet) boundary conditions are set. Stimulation is carried out by injecting a $1 \mathrm{~ms}$ duration current pulse into the first five elements of the strand at an amplitude of $100 \mu \mathrm{A} \mathrm{cm}^{-2}$ ( $\sim 2 \times$-threshold). An implicit integration scheme was also used to check the results presented below; the findings were the same, with a small shift in the exact value of $\left[\mathrm{K}^{+}\right]_{o}$ at which a particular rhythm is seen. Simulations were carried out on $\mathrm{K} 7$ (Athlon $650 \mathrm{MHz}$ ) machines using programs written in $\mathrm{C}(\sim 16$ significant decimal places).

\section{RESULTS}

\section{A. Effect of discretization}

We first investigate the effect of changing the spacing of the numerical grid on the propagated action potential in the homogeneous one-dimensional cable at the nominal $\left[\mathrm{K}^{+}\right]_{o}$ of $5.4 \mathrm{mM}$. The cable is allowed to rest for $1000 \mathrm{~ms}$, and then stimulation is started at a basic cycle length (BCL) of $400 \mathrm{~ms}$. Figure 1(a) shows the upstroke of the 21st action potential at $\Delta x=0.005 \mathrm{~cm}, 0.01 \mathrm{~cm}$, and $0.025 \mathrm{~cm}$ at the mid-point of a $5 \mathrm{~cm}$ long cable with $\Delta t=0.005 \mathrm{~ms}, 0.01 \mathrm{~ms}$, and $0.025 \mathrm{~ms}$, respectively. At $(\Delta x, \Delta t)$ $=(0.025 \mathrm{~cm}, 0.025 \mathrm{~ms})$, where the discretization factor $(\Delta x, \lambda)$ is 0.4 , there is an oscillation following the upstroke, which is much less pronounced at $(\Delta x, \Delta t)$ $=(0.01 \mathrm{~cm}, 0.01 \mathrm{~ms})$ and $(\Delta x, \Delta t)=(0.005 \mathrm{~cm}, 0.005 \mathrm{~ms})$. A similar oscillation has been described previously in the Beeler-Reuter model when the discretization factor is too large. ${ }^{56,57}$ The two upstrokes for $(\Delta x, \Delta t)$ $=(0.01 \mathrm{~cm}, 0.01 \mathrm{~ms})$ and $(\Delta x, \Delta t)=(0.005 \mathrm{~cm}, 0.005 \mathrm{~ms})$ look very similar in Fig. 1(a). In fact, Figs. 1(b)-1(e) show that several action potential parameters are within a few percent of their asymptotic (i.e., $\Delta x / \lambda \rightarrow 0)$ values at $(\Delta x, \Delta t)$ $=(0.01 \mathrm{~cm}, 0.01 \mathrm{~ms})$, which agrees with prior work on the unmodified LR equations. ${ }^{58}$ For example, decreasing $(\Delta x, \Delta t)$ from $(0.01 \mathrm{~cm}, 0.01 \mathrm{~ms})$ to $(0.005 \mathrm{~cm}, 0.005 \mathrm{~ms})$ changes the maximum upstroke velocity $\left(d V / d t_{\max }\right.$ or $\left.\dot{V}_{\max }\right)$ by $7 \%$, the conduction velocity $(\nu)$ by $4 \%$, the maximum voltage (overshoot potential in this case) $\left(V_{\max }\right)$ by $3 \%$, and the action potential duration (APD) by $0.004 \%$. We therefore

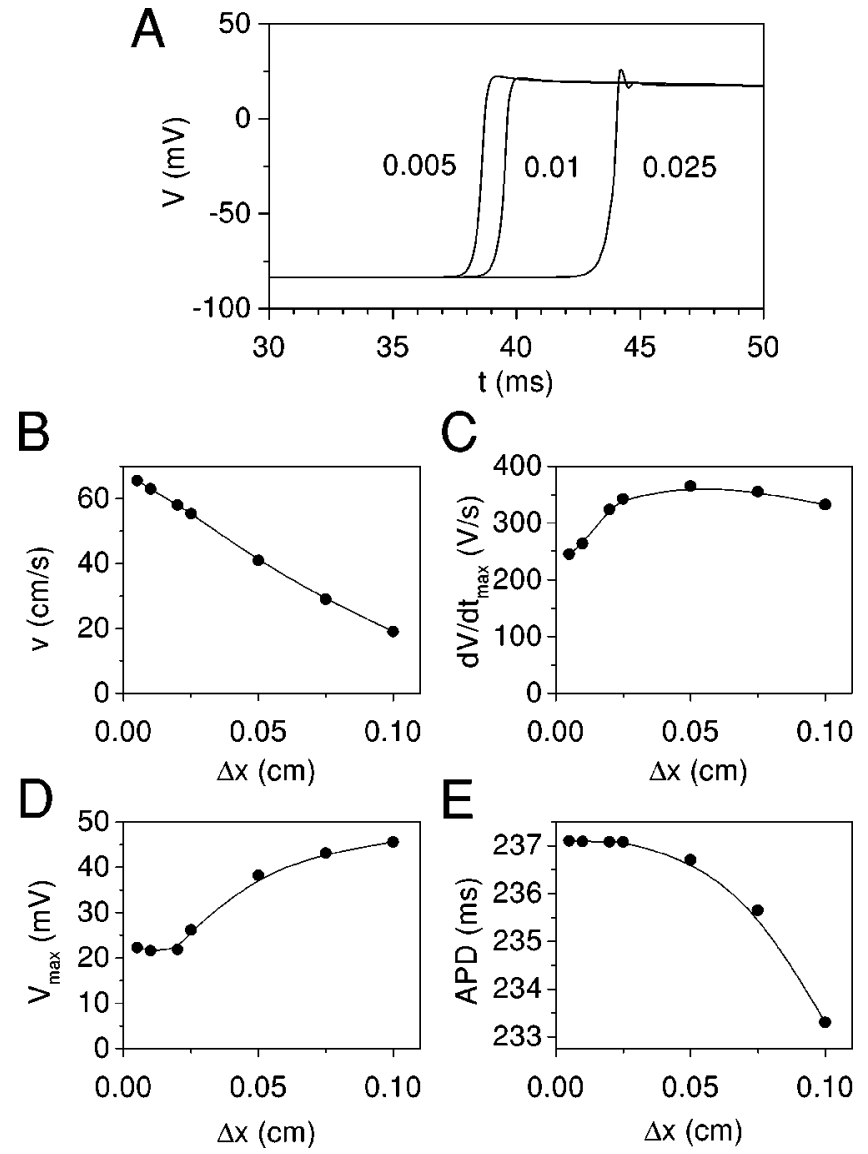

FIG. 1. Action potential propagation in homogeneous one-dimensional cable at $\left[\mathrm{K}^{+}\right]_{o}=5.4 \mathrm{mM}$. (a) Action potential upstrokes at middle of a $5 \mathrm{~cm}$ long cable for three different values of the spatial integration step-size $(\Delta x)$ and the temporal integration step-size $(\Delta t) . \Delta x=0.005 \mathrm{~cm}, \Delta t$ $=0.005 \mathrm{~ms} ; \Delta x=0.01 \mathrm{~cm}, \Delta t=0.01 \mathrm{~ms} ; \Delta x=0.025 \mathrm{~cm}$ at $\Delta t=0.025 \mathrm{~ms}$. The $21 \mathrm{st}$ action potential after the start of stimulation from infinite-rest initial conditions (stimulus pulse amplitude $=100 \mu \mathrm{A} \mathrm{cm}^{-2}$; pulse duration $=1 \mathrm{~ms}$ ). (b)-(e) Effect of changing $\Delta t$ and $\Delta x$ on conduction velocity $(v)$, maximal upstroke velocity $\left(d V / d t_{\max }\right.$ or $\left.\dot{V}_{\max }\right)$, maximum potential $\left(V_{\max }\right)$, and action potential duration (APD). The numerical value of $\Delta t$ (in $\mathrm{ms}$ ) equals the numerical value of $\Delta x$ (in $\mathrm{cm}$ ). $v$ measured between $x=2.5 \mathrm{~cm}$ and $x=3.0 \mathrm{~cm}$ in the cable; other parameters measured at $x=2.5 \mathrm{~cm}$. Curves through the data points are spline fits.

use $\Delta t=0.01 \mathrm{~ms}$ and $\Delta x=0.01 \mathrm{~cm}$ in what follows. The maximum change in $V$ in a simulation from time $t$ to time $t+\Delta t$ is then $2.6 \mathrm{mV}$.

\section{B. Effect on rhythm of increasing $\left[\mathrm{K}^{+}\right]_{o}$ in the ischemic zone}

We model the effect of ischemia by increasing $\left[\mathrm{K}^{+}\right]_{o}$ in the central part of the strand (the ischemic zone). ${ }^{1,28,37,40-43,59}$ We choose a fixed BCL of $400 \mathrm{~ms}$, which is within the range used in experimental work on ischemic arrhythmias (see Ref. 1 for references). The total length of the strand is $3.0 \mathrm{~cm}$, with the region of elevated $\left[\mathrm{K}^{+}\right]_{o}$ occupying the central 1.0 $\mathrm{cm}$ length of the strand, to correspond with our earlier simulations in a two-dimensional sheet. ${ }^{1}$ At the start of each simulation run at a given $\left[\mathrm{K}^{+}\right]_{o}$, we obtain approximate infiniterest initial conditions by setting the variables in the normaland high-potassium regions equal to their respective space- 

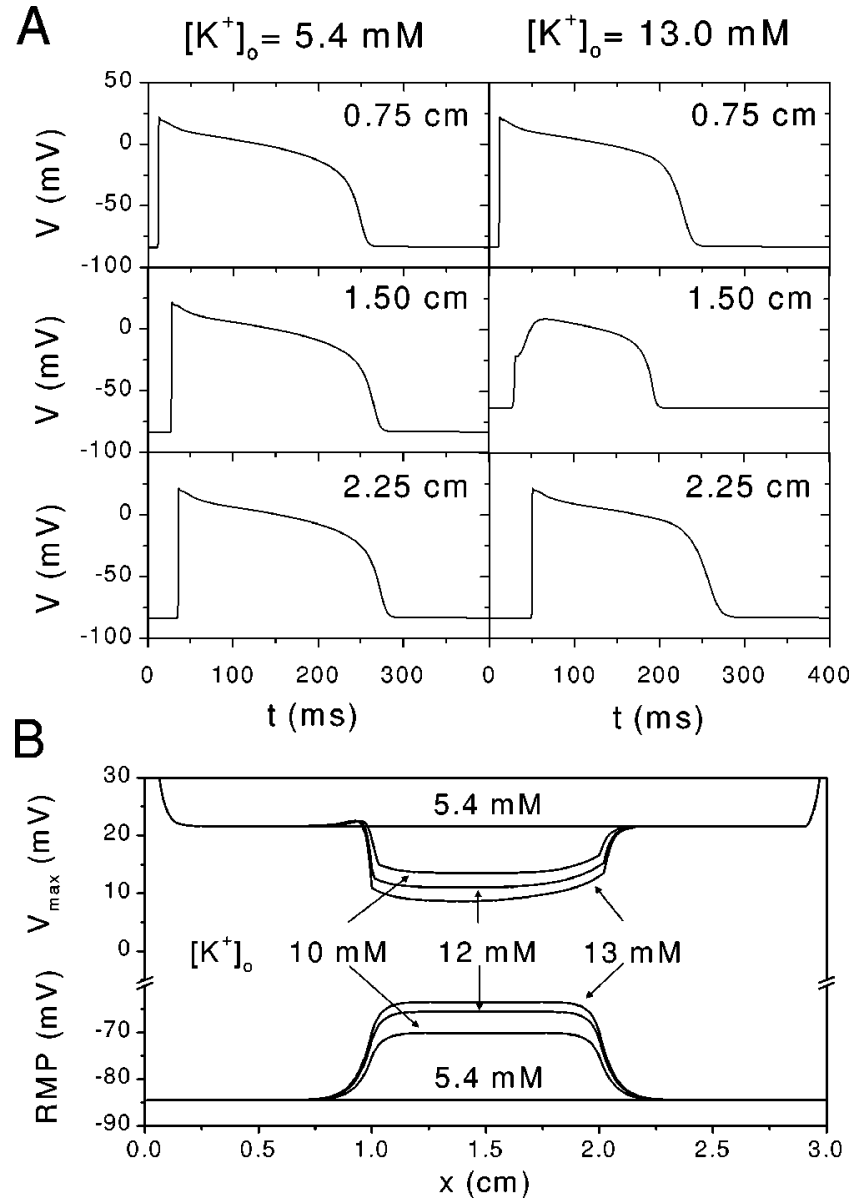

FIG. 2. Action potential propagation during 1:1 rhythm in the onedimensional cable. (a) Action potentials at three sites: proximal normal zone (top: $x=0.75 \mathrm{~cm}$ ), middle of ischemic zone (middle: $x=1.5 \mathrm{~cm}$ ), distal normal zone (bottom: $x=2.25 \mathrm{~cm}) .\left[\mathrm{K}^{+}\right]_{o}=5.4 \mathrm{mM}($ left $)$ and $13.0 \mathrm{mM}($ right $)$. (b) Resting membrane potential (RMP) and maximum potential $\left(V_{\max }\right)$ as a function of distance $(x)$ during a 1:1 rhythm. The ischemic zone lies between $x=1 \mathrm{~cm}$ and $x=2 \mathrm{~cm}$.

clamped steady-state values and then allowing the simulation to run for $1000 \mathrm{~ms}$, so as to allow some time for equilibration to occur before injecting the first stimulus at $t=0 \mathrm{~ms}$. We now describe the sequence of rhythms seen as $\left[\mathrm{K}^{+}\right]_{o}$ is raised.

Figure 2(a) (left) shows action potentials recorded at three locations $(x=0.75,1.5$, and $2.25 \mathrm{~cm})$ in the strand, with $\left[\mathrm{K}^{+}\right]_{o}$ set to its nominal value of $5.4 \mathrm{mM}$ (i.e., in the control situation, before the onset of simulated ischemia). There is a 1:1 response at these (and all other) sites in the strand, in that each stimulus produces an action potential of essentially invariant morphology (neglecting edge effects) that propagates down the entire length of the strand, at a conduction velocity of $63 \mathrm{~cm} \mathrm{~s}^{-1}$. As $\left[\mathrm{K}^{+}\right]_{o}$ is raised [e.g., Fig. 2(a), right: $\left.\left[\mathrm{K}^{+}\right]_{o}=13.0 \mathrm{mM}\right]$, the $1: 1$ response is preserved, but there is a progressive depolarization of the resting membrane potential (RMP) and decrease in the maximum potential $\left(V_{\max }\right)$ obtained as the action potential enters the ischemic zone [Fig. 2(b)]. There are also decreases in $\dot{V}_{\max }$, APD, and $\nu$ within the ischemic zone [see, e.g., Fig. 2(a), right: $x$ $=1.5 \mathrm{~cm}]$. The $\mathrm{K}^{+}$-induced decreases in $V_{\max }, \dot{V}_{\max }$, and $\nu$ which have been described previously in ventricular ionic

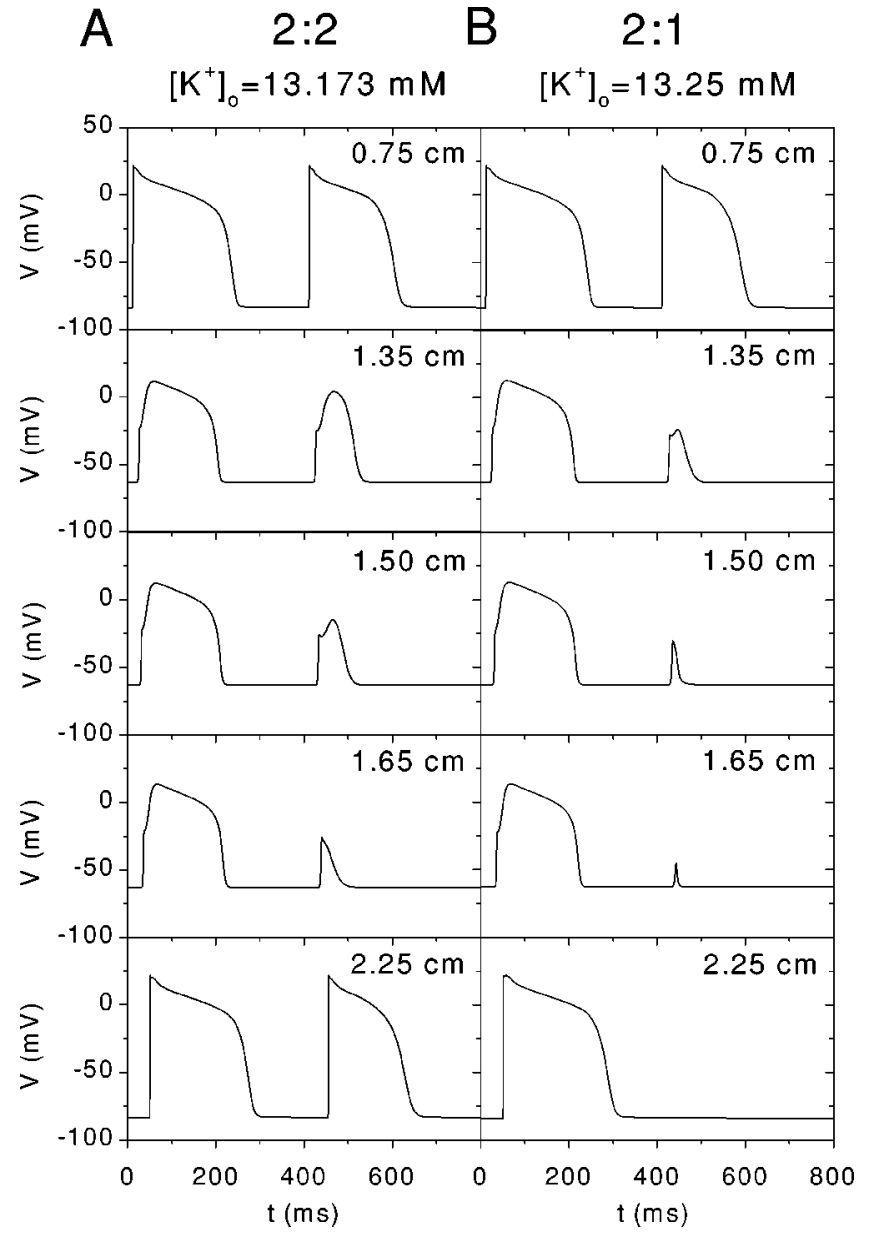

FIG. 3. Action potential propagation during 2:2 and 2:1 rhythms in the one-dimensional cable. Action potentials at five sites in the one-dimensional cable during: (a) $2: 2$ rhythm $\left(\left[\mathrm{K}^{+}\right]_{o}=13.173 \mathrm{mM}\right)$, (b) $2: 1$ rhythm $\left(\left[\mathrm{K}^{+}\right]_{o}\right.$ $=13.25 \mathrm{mM}$ ).

models, ${ }^{1,28,37,40-44,59}$ are due to a reduction in the fast inward sodium current $\left(I_{\mathrm{Na}}\right)$ caused by the $\mathrm{K}^{+}$-induced depolarization of the resting potential, while the reduction in APD is largely due to the $\mathrm{K}^{+}$-activation of the maximal conductances of the outward repolarizing $\mathrm{K}^{+}$currents. While the action potential thus decrements as it enters the ischemic zone, it increments as it leaves, with the degree of initial decrement and subsequent increment increasing as $\left[\mathrm{K}^{+}\right]_{0}$ is raised [Fig. 2(b)].

As $\left[\mathrm{K}^{+}\right]_{o}$ is increased further, there is eventually a loss of 1:1 synchronization, with a transition to an alternans or $2: 2$ rhythm, in which there is a beat-to-beat alternation in the morphology of the action potential between two different morphologies [e.g., Fig. 3(a): $\left.\left[\mathrm{K}^{+}\right]_{o}=13.173 \mathrm{mM}\right]$. The smaller action potential in Fig. 3(a) asymptotes to a small size (amplitude $\cong 30 \mathrm{mV}$ ) towards the distal end of the ischemic zone (see, e.g., $x=1.65 \mathrm{~cm}$ trace), but then increments back up to a normal size $(x=2.25 \mathrm{~cm}$ trace $)$ as it leaves the ischemic zone. Figure 4 (top three traces) shows $V_{\text {max }}$ as a function of position for the smaller action potential during alternans at three different values of $\left[\mathrm{K}^{+}\right]_{o}(13.173$, 13.183 , and $13.2 \mathrm{mM}$ ). This smaller action potential propagates with constant velocity over a considerable part of the distal ischemic zone, with $V_{\max }$ staying constant (e.g., over a 


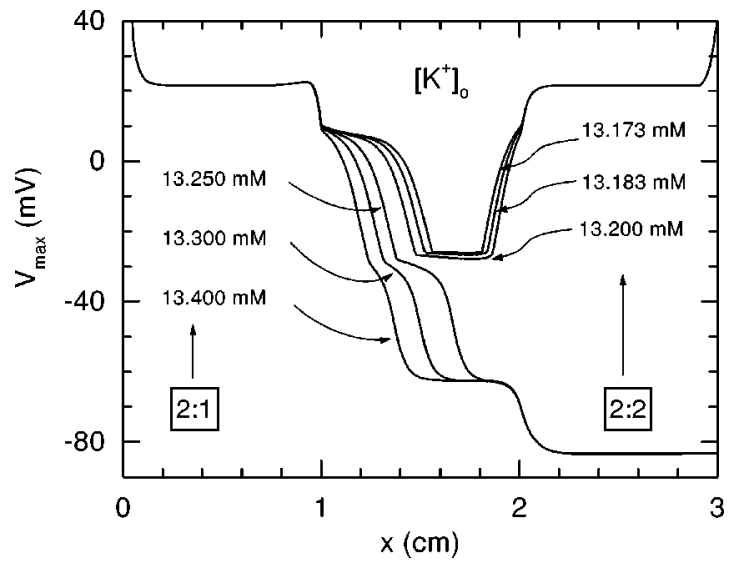

FIG. 4. Maximum potential as a function of distance. Maximum potential $\left(V_{\max }\right)$ as a function of distance $(x)$ for the smaller of the two responses during 2:2 rhythm (upper three traces: $\left[\mathrm{K}^{+}\right]_{o}=13.173,13.183$, and 13.2 $\mathrm{mM}$ ) and 2:1 rhythm (lower three traces: $\left[\mathrm{K}^{+}\right]_{o}=13.25,13.3$, and 13.4 $\mathrm{mM})$. For a $2: 1$ rhythm (lower three traces), when $x$ is sufficiently large, $V_{\max }$ equals the resting membrane potential.

distance of $\sim 0.25 \mathrm{~cm}$ at $\left[\mathrm{K}^{+}\right]_{o}=13.173 \mathrm{mM}$ in Fig. 4). However, over this part of the ischemic zone, the APD first decreases slightly and then increases, due to the close spatial proximity of regions of decremental and incremental conduction, respectively. When sufficient space for propagation is available (e.g., in a $3 \mathrm{~cm}$ homogeneous cable with $\left[\mathrm{K}^{+}\right]_{o}$ $=13.2 \mathrm{mM}$ everywhere), this type of very small-amplitude action potential asymptotes towards an invariant waveform [similar to that seen at $x=1.65 \mathrm{~cm}$ in Fig. 3(a)] as it proceeds down the cable. Very similar convergent behavior is also seen in a homogeneous cable at $\left[\mathrm{K}^{+}\right]_{o}=5.4 \mathrm{mM}$ when $I_{s}$ is set to zero and $I_{\mathrm{Na}}$ reduced to a value just above the critical value that supports a propagating response. This response is thus a genuine propagating response, which we have previously termed the "maintained small-amplitude response." 1 With an increase in $\left[\mathrm{K}^{+}\right]_{o}$, the smaller action potential of the 2:2 rhythm attains its asymptotic value of $V_{\max }$ earlier within the ischemic zone, and the small-amplitude response recovers back to a full-sized action potential later as it leaves the ischemic zone (see $V_{\max }$ traces at $\left[\mathrm{K}^{+}\right]_{o}=13.173$, 13.183, and $13.2 \mathrm{mM}$ in Fig. 4). Note that at a given $\left[\mathrm{K}^{+}\right]_{o}$ the degree of alternation decreases as one moves away from the center of the ischemic zone and out towards either end of the cable [Fig. 3(a)].

With a still further increase in $\left[\mathrm{K}^{+}\right]_{o}$, there comes the point where the smaller action potential does not turn into a maintained small-amplitude response within the ischemic zone; instead, it decrements sharply within the proximal part of the ischemic zone, and eventually dies out within the ischemic zone [Fig. 3(b): $\left.\left[\mathrm{K}^{+}\right]_{o}=13.25 \mathrm{mM}\right]$, resulting in a 2:1 response within the distal portion of the ischemic zone and throughout the entire distal normal zone. In the part of the strand proximal to the area of block, there is a $2: 2$ rhythm present [e.g., Fig. 3(b): $x=0.75,1.35 \mathrm{~cm}]$. As $\left[\mathrm{K}^{+}\right]_{o}$ is increased during $2: 1$ rhythm, the action potential that is eventually blocked suffers a greater rate of decrement within the proximal part of the ischemic zone, so that the site of block gradually moves to a more proximal location (Fig. 4:

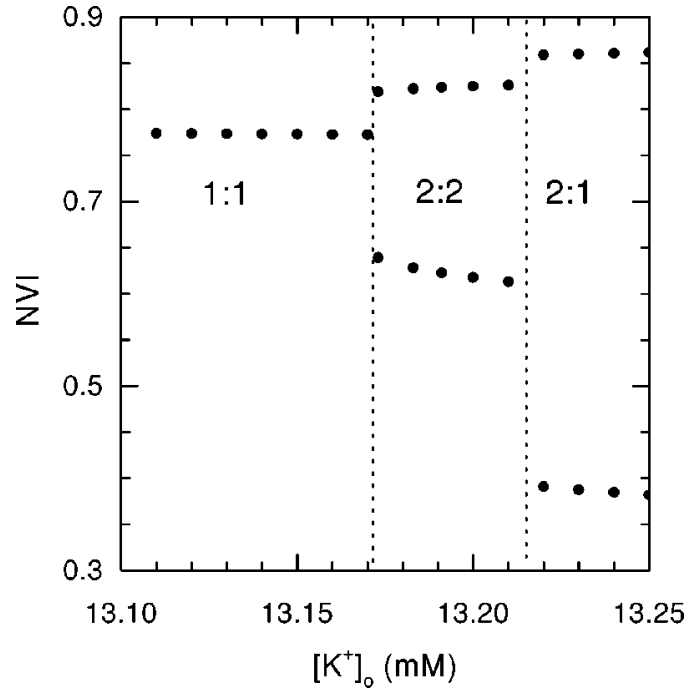

FIG. 5. Bifurcation diagram. The normalized voltage integral (NVI) is plotted as a function of the bifurcation parameter $\left(\left[\mathrm{K}^{+}\right]_{o}\right)$. The 2:1 rhythm is seen until $\left[\mathrm{K}^{+}\right]_{o}=13.4 \mathrm{mM}$

$\left[\mathrm{K}^{+}\right]_{o}=13.25,13.30$, and $\left.13.40 \mathrm{mM}\right)$. A still further increase in $\left[\mathrm{K}^{+}\right]_{o}$ results in period- 4 , period- 6 , and period- 8 rhythms, and eventually the appearance of subthreshold rhythms containing no action potentials (i.e., complete block) at $\left[\mathrm{K}^{+}\right]_{o}$ $\cong 13.6 \mathrm{mM}$. The sequence of rhythms seen is $\{1: 1 \rightarrow 2: 2$ $\rightarrow 2: 1 \rightarrow 4: 2 \rightarrow 4: 1 \rightarrow 6: 2 \rightarrow 6: 1 \rightarrow 8: 2 \rightarrow 8: 1 \rightarrow 2: 0 \rightarrow 1: 0\}$.

\section{Bifurcation diagram}

One usually constructs a one-parameter bifurcation diagram by plotting the steady-state value of some variable (or the maximum and/or minimum value of that variable) as a function of the bifurcation parameter $\left(\left[\mathrm{K}^{+}\right]_{o}\right.$ in our case $)$. However, the choice of such a variable to act as an index of the state of the system is not obvious when one has a spatially distributed system (such as we have here), since one would like to have the activity seen at all of the spatial locations contribute to that index. We thus introduce as our bifurcation index the normalized voltage integral (NVI), which represents the voltage averaged over space and time during the course of one $400 \mathrm{~ms}$ stimulation cycle. The spatial averaging is obtained by adding together the voltages at all 300 grid-points at a given time-step. The temporal averaging is then obtained by adding together these sums for every fifth integration time-step (i.e., every $0.05 \mathrm{~ms}$ ) during each individual $400 \mathrm{~ms}$ stimulation cycle. The resulting sum of sums is the voltage integral. The normalized voltage integral is then obtained by dividing this voltage integral by the voltage integral obtained at $\left[\mathrm{K}^{+}\right]_{o}=5.4 \mathrm{mM}$.

The bifurcation diagram in Fig. 5, in which we plot NVI as a function of $\left[\mathrm{K}^{+}\right]_{o}$, summarizes the results described above, showing the transition from $1: 1$ to $2: 2$ rhythm, and then from $2: 2$ to $2: 1$ rhythm, as $\left[\mathrm{K}^{+}\right]_{o}$ is increased. The data points with $\mathrm{NVI} \cong 0.6$ during $2: 2$ rhythm correspond to the maintained small-amplitude response seen during that rhythm [Figs. 3(a), 4]. Note also the increase in the value of the integral for the larger beat upon the transition from $2: 2$ to 
$2: 1$ rhythm, with $\mathrm{NVI} \cong 0.8$ corresponding to this propagated

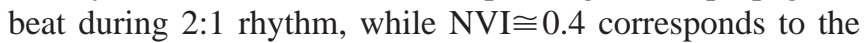
blocked beat [Figs. 3(b), 4].

\section{Reduction to a one-dimensional map}

In clinical, ${ }^{60,61}$ experimental, ${ }^{32-35,62-67}$ and modeling $22-24,26,27,42,68$ work, it has been shown that analysis of the response of a cardiac preparation to periodic stimulation can often be reduced to consideration of a one- or twodimensional finite-difference equation ("map"). One of two different approaches is usually taken to extract a onedimensional map. In the first method, one observes an irregular rhythm during steady-state conditions and simply plots some index of the activity on any given beat (e.g., APD) as a function of the value of that index on the preceding beat. In the second method, one applies an S1S2 premature stimulation protocol during 1:1 rhythm, characterizes the premature beat (e.g., by its APD) as a function of the S1S2 coupling interval, and then, using certain assumptions, writes down a one-dimensional map that can be iterated to then predict the response of the preparation to periodic driving at any BCL.

Here we use a combination of the above two stimulation protocols to extract a map. At a given $\left[\mathrm{K}^{+}\right]_{o}$, from infiniterest initial conditions, we apply two $\mathrm{S} 1$ stimuli at an S1S1 interval of $400 \mathrm{~ms}$, and then inject a single pre- or postmature S2 stimulus at a variable S1S2 coupling interval (range 300-800 ms). Following the S2 stimulus, we then apply 30 S3 stimuli at our standard BCL of $400 \mathrm{~ms}$. The effect of the S1S2 premature stimulation protocol is thus to change the initial conditions from which periodic pacing at $\mathrm{S} 3 \mathrm{~S} 3=400 \mathrm{~ms}$ is started. At each S1S2 interval we then calculate the voltage integral for each of the $30 \mathrm{~S} 3$ beats following the S2 stimulus, and plot each normalized voltage integral $\left(\mathrm{NVI}_{i+1}\right)$ as a function of the immediately preceding integral $\left(\mathrm{NVI}_{i}\right)$. We shall refer to this map as the "voltage integral map."

Figure 6(a) illustrates the voltage integral map obtained at $\left[\mathrm{K}^{+}\right]_{o}=13.0 \mathrm{mM}$ with this $\mathrm{S} 1 \mathrm{~S} 2$ protocol, where a $1: 1$ rhythm is seen [Fig. 2(a), right]. A pre- or post-mature S2 stimulus results in a transient episode of alternans, before $1: 1$ rhythm is re-established asymptotically. This results in the map having a negative slope. There is a stable period-1 fixed point on this map at $\mathrm{NVI} \cong 0.78$, which, in the simulations, corresponds to a 1:1 rhythm involving propagation of a large-sized action potential, as in Fig. 2(a) (right).

There is a gap in the map for $\sim 0.33<\mathrm{NVI}_{i}<\sim 0.52$. The data point just to the left of this gap is produced when S1S2 is quite short, and corresponds to an action potential that just barely fails to exit the ischemic zone successfully $\left(\mathrm{NVI}_{i} \cong 0.33\right)$ being followed by a full-sized action potential $\left(\mathrm{NVI}_{i+1} \cong 0.86\right)$. The point just to the right of the gap is produced at a slightly longer S1S2 interval, when an action potential that just barely manages to exit the ischemic zone $\left(\mathrm{NVI}_{i} \cong 0.52\right)$ is followed by a full-sized action potential $\left(\mathrm{NVI}_{i+1} \cong 0.83\right)$. An increase in $\mathrm{S} 1 \mathrm{~S} 2$ of $0.01 \mathrm{~ms}$ (the smallest increment we have used) is sufficient to convert an action potential that blocks at the very distal end of the ischemic zone into one that successfully propagates into and through
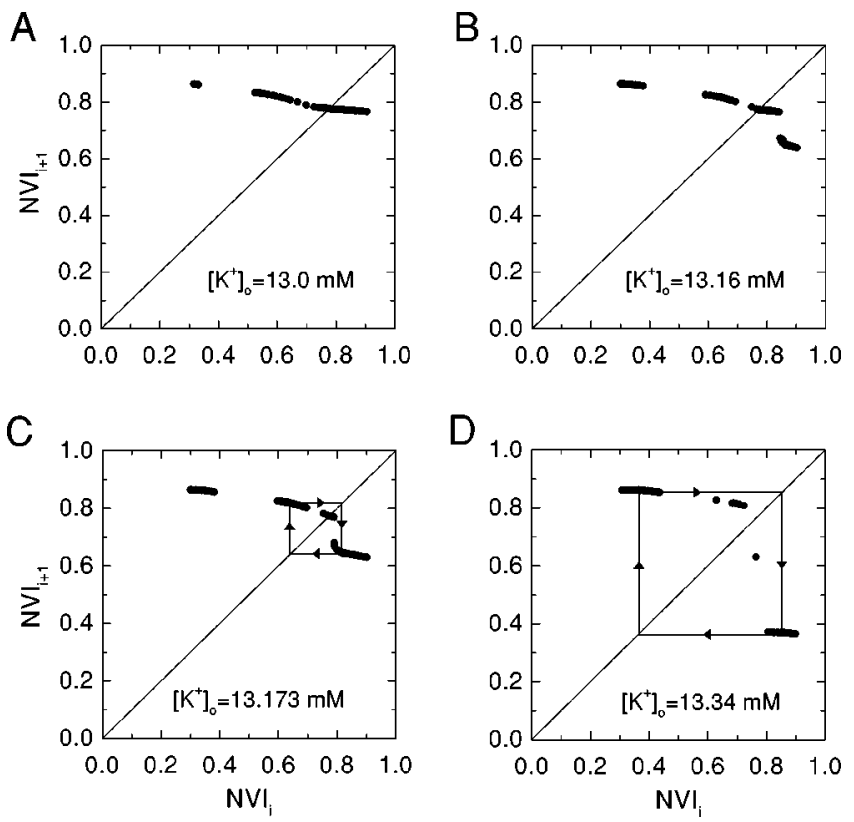

FIG. 6. One-dimensional maps at 4 different values of $\left[\mathrm{K}^{+}\right]_{o}$. Maps produced by an S1S2 stimulation protocol (S1S2 $=300-800 \mathrm{~ms})$, as described in the text. (a) $\left[\mathrm{K}^{+}\right]_{o}=13.0 \mathrm{mM}$. The map has only one branch. There is a stable period-1 orbit, corresponding to a $1: 1$ rhythm. (b) $\left[\mathrm{K}^{+}\right]_{o}$ $=13.16 \mathrm{mM}$. A second branch of the map can now be seen at the right. There is still a stable period-1 orbit present, corresponding to $1: 1$ rhythm. There is no period-2 orbit. (c) $\left[\mathrm{K}^{+}\right]_{o}=13.173 \mathrm{mM}$. As well as the stable period-1 orbit (corresponding to 1:1 rhythm), there is also now present a stable period-2 orbit (arrowheads), corresponding to 2:2 rhythm. (d) $\left[\mathrm{K}^{+}\right]_{o}=13.34 \mathrm{mM}$. There is no longer a stable period-1 orbit present. Rather there is now a stable period-2 orbit (arrowheads), corresponding to $2: 1$ rhythm.

the distal normal zone. Thus we have no data points for $\sim 0.33<\mathrm{NVI}_{i}<\sim 0.52$.

As $\left[\mathrm{K}^{+}\right]_{o}$ is increased, the map moves downwards and to the left, so that the fixed point moves to a lower value; in the simulations, this corresponds to a fall in NVI as $\left[\mathrm{K}^{+}\right]_{o}$ is increased with $1: 1$ rhythm being maintained, which is largely due to the decrease in the amplitude and duration of the action potential within the ischemic zone [Fig. 2(b)].

As $\left[\mathrm{K}^{+}\right]_{o}$ is increased further, a second branch eventually appears lower down on the map to the right [e.g., Fig. 6(b): $\left.\left[\mathrm{K}^{+}\right]_{o}=13.16 \mathrm{mM}\right]$, but there remains a period-1 orbit corresponding to $1: 1$ rhythm. With our degree of precision (changing the $\mathrm{S} 1 \mathrm{~S} 2$ coupling interval in steps as fine as 0.01 $\mathrm{ms}$ ), the map effectively has a discontinuity between the two branches [at $\mathrm{NVI}_{i} \cong 0.84$ in Fig. 6(b)]. Points $\left(\mathrm{NVI}_{i}\right.$, $\mathrm{NVI}_{i+1}$ ) lying on the right-hand branch in Fig. 6(b) all have $\mathrm{NVI}_{i}>0.84$ and $\mathrm{NVI}_{i+1} \cong 0.64-0.67$, corresponding to the fact that $\left[\mathrm{K}^{+}\right]_{o}$ is now so high that a sufficiently large action potential $\left(\mathrm{NVI}_{i}>0.84\right)$ is now followed by so short a recovery time that the following response is only the maintained small-amplitude response $\left(\mathrm{NVI}_{i+1} \cong 0.64-0.67\right.$ - see the smaller of the two responses of 2:2 rhythm in Fig. 5). In contrast, should $\mathrm{NVI}_{i}$ be sufficiently small $\left(\mathrm{NVI}_{i}<0.84\right)$, one lands on the left-hand branch, corresponding in the simulations to a full-sized action potential $\left(\mathrm{NVI}_{i+1}\right.$ $\cong 0.76-0.86$ ) being preceded by either a blocked beat $\left(\mathrm{NVI}_{i} \cong 0.2-0.4\right)$, a maintained small-amplitude response 
$\left(\mathrm{NVI}_{i} \cong 0.6-0.7\right)$, or a full-sized action potential $\left(\mathrm{NVI}_{i}\right.$ $\cong 0.75-0.84$ ). The slope of the right-hand branch of the map is also negative, for a reason similar to that outlined above for the left-hand branch: i.e., a larger action potential $\left(\mathrm{NVI}_{i}\right)$ on this branch will be followed by a smaller maintained small-amplitude response $\left(\mathrm{NVI}_{i+1}\right)$. As $\left[\mathrm{K}^{+}\right]_{o}$ is increased, the right-hand branch moves down and to the left, so that the point of (effective) discontinuity in the map also moves to the left.

At $\left[\mathrm{K}^{+}\right]_{o}=13.173 \mathrm{mM}$, the map admits a stable period-2 orbit, as well as the period-1 orbit just described above [Fig. 6(c)]. This period-2 orbit corresponds to an alternans rhythm consisting of a maintained small-amplitude response $(\mathrm{NVI} \cong 0.65)$ alternating with a full-sized action potential ( $\mathrm{NVI} \cong 0.82$ ). Each of the period-1 and period-2 orbits has its own basin of attraction. As $\left[\mathrm{K}^{+}\right]_{o}$ is slightly increased, the period-1 orbit disappears, leaving only the period-2 orbit corresponding to 2:2 rhythm.

For sufficiently high $\left[\mathrm{K}^{+}\right]_{o}$, the right-hand branch of the map is found to lie much lower down and there is a period-2 orbit involving this branch [Fig. $\left.6(\mathrm{~d}):\left[\mathrm{K}^{+}\right]_{o}=13.34 \mathrm{mM}\right]$. This period-2 orbit corresponds to a 2:1 rhythm consisting of a full-sized action potential $(\mathrm{NVI} \cong 0.86)$ alternating with a blocked beat $(\mathrm{NVI} \cong 0.37)$ - see Figs. 4 and 5 . This very abrupt transition from 2:2 rhythm to 2:1 rhythm occurs when the incremental propagation that turns the maintained smallamplitude response into a full-sized action potential during 2:2 rhythm [Figs. 3(a), 4] becomes decremental conduction upon exiting the ischemic zone. The abruptness of this transition can be appreciated from the fact that decreasing the coupling interval by only $0.01 \mathrm{~ms}$ in an S1S2 premature stimulation protocol suffices to convert an incrementing into a decrementing response at $\left[\mathrm{K}^{+}\right]_{o}=13.0 \mathrm{mM}$, producing effectively all-or-none propagation.

\section{E. $\{1: 1 \leftrightarrow 2: 2\}$ bistability}

In all of the numerical simulation results presented above (Figs. 2-5), each simulation run was started from only one set of initial conditions. However, the map analysis above predicts that bistability should occur [Fig. 6(c)]. Figure 7 shows that dropping a single stimulus from the periodic drive train at that exact value of $\left[\mathrm{K}^{+}\right]_{o}(13.173 \mathrm{mM})$ does indeed result in the conversion of the $1: 1$ rhythm into a $2: 2$ rhythm, demonstrating the existence of $\{1: 1 \leftrightarrow 2: 2\}$ bistability. In addition, the reverse transition from 2:2 to $1: 1$ rhythm can be induced by the injection of a suitably timed extrastimulus.

\section{F. Piecewise-linear map}

Our simulations (Figs. 2-5, 7) and the NVI maps (Fig. $6)$ indicate that the sequence of rhythms $\{1: 1$ alone $\rightarrow 1: 1$ coexisting with $2: 2 \rightarrow 2: 2$ alone $\rightarrow 2: 1\}$ is seen as $\left[\mathrm{K}^{+}\right]_{o}$ is increased. We decided to explore the bifurcation sequence systematically using a piecewise-linear map that is an approximation to the maps in Figs. 6(a)-6(c). For $\left[\mathrm{K}^{+}\right]_{o}$ sufficiently low, the map has a single branch, which is the straight line $\mathrm{NVI}_{i+1}=-0.2\left(\mathrm{NVI}_{i}\right)+0.9$ [Fig. 8(a)], which has a negative slope that is $<1$ in absolute value. There is

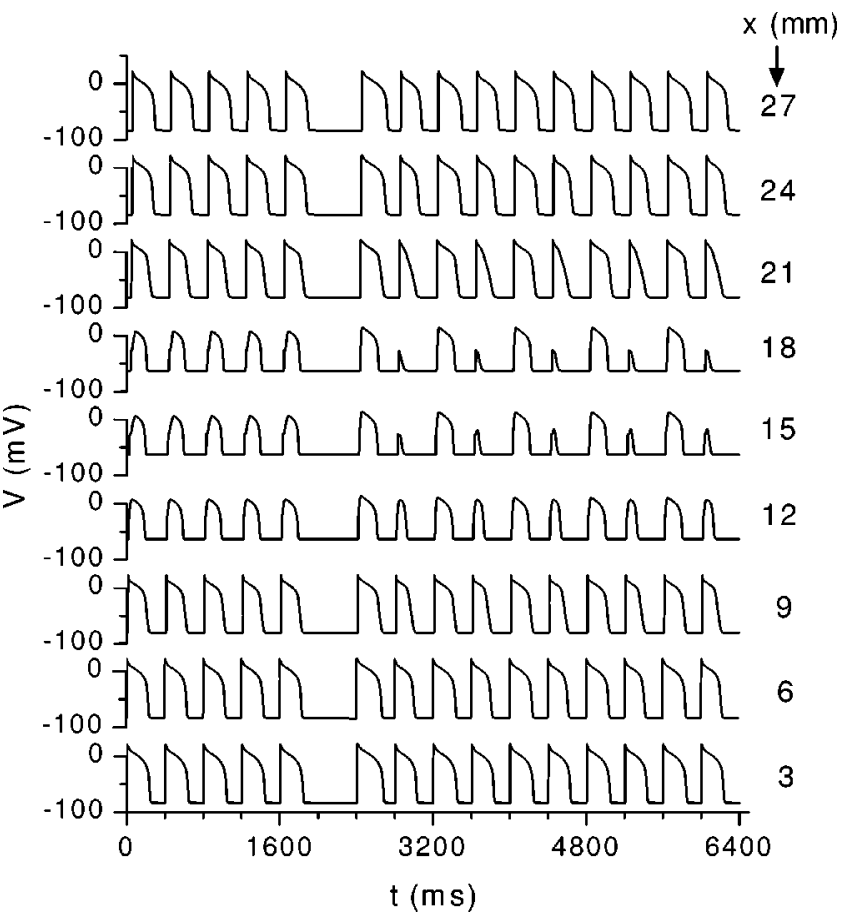

FIG. 7. Conversion from 1:1 to 2:2 rhythm caused by dropping one stimulus from the periodic drive train. The 1:1 rhythm existed for 100 cycles before the stimulus was dropped, and the 2:2 rhythm persisted for 100 stimuli (the length of the longest integration run that we made) following the resumption of stimulation after the stimulus was dropped.

therefore a globally-attracting period-1 orbit on this map, corresponding to 1:1 rhythm [compare Fig. 8(a) with Fig. 6(a) $]$. As $\left[\mathrm{K}^{+}\right]_{o}$ is increased, a second branch, with the same slope, makes its appearance [Fig. 8(b)]. The equation of this branch is $\mathrm{NVI}_{i+1}=-0.2\left(\mathrm{NVI}_{i}\right)+0.8$, so that there is a jump discontinuity of size 0.1 between the two branches of the map [compare Fig. 8(b) with Fig. 6(b)]. Nevertheless, the period-1 orbit remains globally attracting. An increase in $\left[\mathrm{K}^{+}\right]_{o}$ corresponds to gradually moving the position of the jump discontinuity (at $\mathrm{NVI}_{i}=\alpha$ ) to the left, leaving the size of the jump unchanged [see also Figs. 6(b) and 6(c)]. There eventually comes a point where a period- 2 orbit appears (corresponding to 2:2 rhythm), which coexists with the period-1 orbit [compare Fig. 8(c) with Fig. 6(c)]. With a still further increase in $\left[\mathrm{K}^{+}\right]_{o}$, and resultant movement to the left in the position of the jump discontinuity (i.e., decrease in $\alpha$ ), the left-hand branch of the map eventually no longer intersects the line of identity, so that the period-1 orbit disappears in a discontinuous fashion, leaving only the period-2 orbit behind [Fig. 8(d)]. Figure 9 gives the bifurcation diagram of the piecewise-linear map, with the bifurcation parameter being the location of the discontinuity $(\alpha)$ in the map. As $\alpha$ decreases (corresponding to an increase of $\left[\mathrm{K}^{+}\right]_{o}$ in the cable simulations), one sees the transition from a period-1 orbit (1:1 rhythm) alone to coexisting period- 1 and period-2 orbits (bistable 1:1 and 2:2 rhythms) and then to a period-2 orbit (2:2 rhythm) alone.

\section{G. Rhythms in space-clamped membrane}

It is obvious that the various rhythms described above in the cable are largely determined by the properties of the 

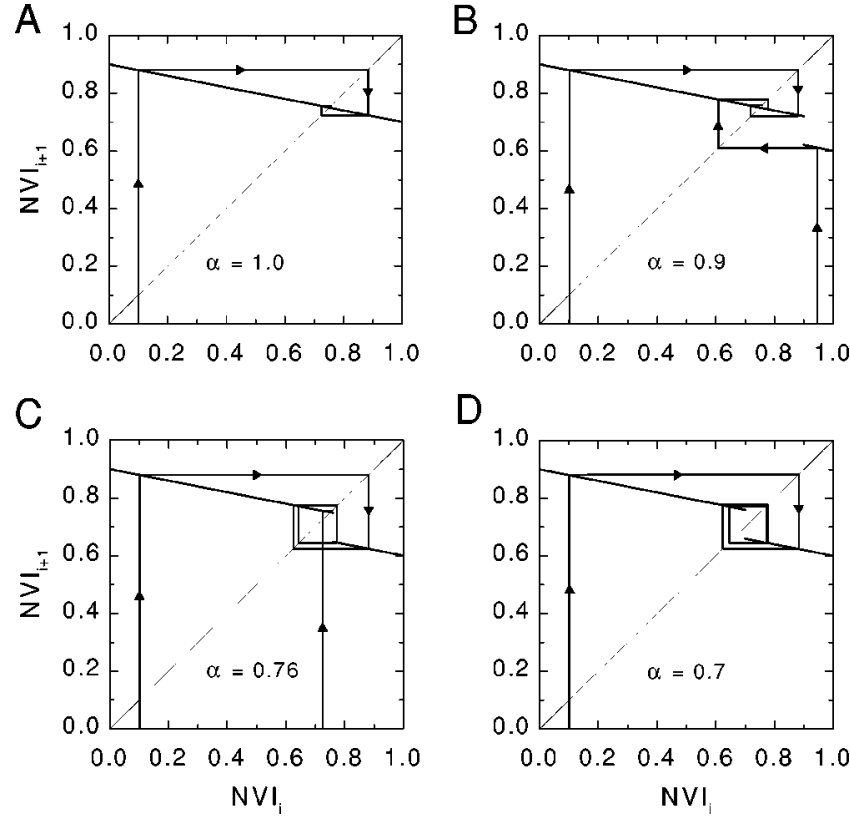

FIG. 8. Piecewise-linear maps approximating the NVI maps from the cable simulations (Fig. 6). (a) There is a globally attracting period-1 orbit, corresponding to $1: 1$ rhythm in the simulations. (b) $\alpha$ (the position of the discontinuity in the map) $=0.9$. Although a second branch now exists on the map, there is still a globally attracting period-1 orbit, corresponding to $1: 1$ rhythm. (c) $\alpha=0.76$. There are now stable period- 1 and period-2 orbits, corresponding to $1: 1$ and $2: 2$ rhythms, respectively. (d) $\alpha=0.70$. The period-1 orbit has disappeared, so that the only stable orbit now present is a period-2 orbit, corresponding to a $2: 2$ rhythm.

$\mathrm{K}^{+}$-depolarized membrane within the central ischemic zone. A question that naturally arises is whether the response of the distributed system (i.e., the one-dimensional cable) to periodic stimulation can then be accounted for by the response of space-clamped $\mathrm{K}^{+}$-depolarized membrane to periodic stimulation. However, this is a very difficult question to investigate systematically for two reasons. First, the waveform of

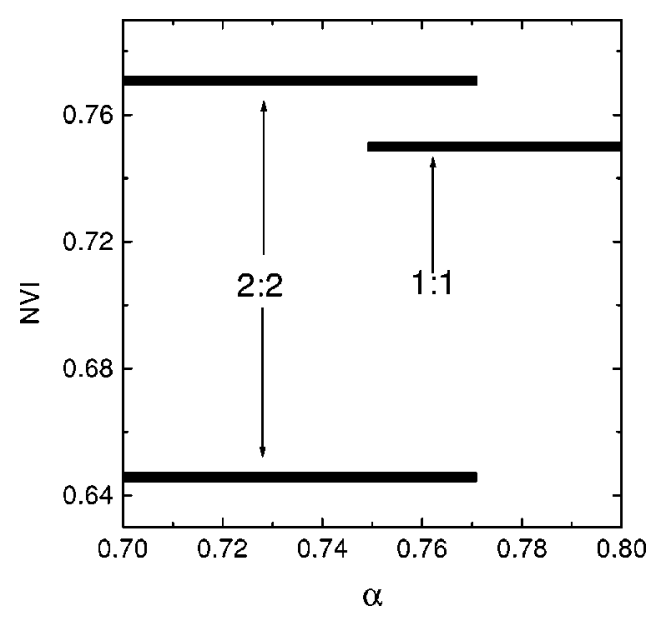

FIG. 9. Bifurcation diagram for the piecewise-linear map. The bifurcation parameter is the position of the discontinuity $(\alpha)$ in the maps of Fig. 8. Note that there is a range of $\alpha$ over which there is bistability between $1: 1$ and 2:2 rhythms. The increment in $\alpha$ is 0.01 . At each value of $\alpha, 100$ iterations were carried out from each of 100 equally-spaced initial conditions. The first 96 iterates were not plotted in each case, to allow time for any transients to pass. the stimulation current entering any particular isopotential grid-element of the cable is very complicated in shape, since the axial or longitudinal current is proportional to $\partial V / \partial x$. Second, this current changes as a function of position along an inhomogeneous cable. For example, during a 2:1 block in the cable, a grid-element lying sufficiently proximal within the ischemic zone will show a local 2:2 response, while an identical grid-element lying sufficiently distal will show a local 2:1 response [Figs. 3(b), 4]. This difference in response must thus be due entirely to the fact that the net longitudinal current (source minus sink) is different in the two gridelements.

In an attempt to investigate this problem, we study the response of the space-clamped membrane to periodic delivery of a $1 \mathrm{~ms}$ duration current-pulse stimulus at a fixed BCL of $400 \mathrm{~ms}$ over a wide range of $\left[\mathrm{K}^{+}\right]_{o}$ and pulse amplitude. At each fixed value of the stimulus amplitude $\left[27 \mu \mathrm{A} \mathrm{cm}^{-2}\right.$ $\left(\sim\right.$ threshold at the nominal $\left[\mathrm{K}^{+}\right]_{o}$ of $\left.5.4 \mathrm{mM}\right)$ to $54 \mu \mathrm{A} \mathrm{cm}^{-2}$, incremented in steps of $\left.1 \mu \mathrm{A} \mathrm{cm}^{-2}\right],\left[\mathrm{K}^{+}\right]_{o}$ is changed in the range $5-20 \mathrm{mM}$. At each pulse amplitude, a $1: 1$ rhythm is seen when $\left[\mathrm{K}^{+}\right]_{o}$ is sufficiently low, and more complex rhythms can appear as $\left[\mathrm{K}^{+}\right]_{o}$ is increased. In particular, a transition from 1:1 to $2: 2$ rhythm can occur, and bistability between these two rhythms can also be seen: e.g., at a stimulus current of $30 \mu \mathrm{A} \mathrm{cm}^{-2}$, which is just above threshold, and $\left[\mathrm{K}^{+}\right]_{o}=14.6677 \mathrm{mM}$, the $1: 1$ rhythm that appears can be converted into a 2:2 rhythm by dropping a stimulus pulse, producing a waveform very similar to that shown at $x=15 \mathrm{~mm}$ in Fig. 7. The similarity of the rhythms and waveforms seen in the space-clamped and distributed cases thus reinforces the conclusion that the local properties of the membrane within the ischemic zone play a key role in determining the overall behavior in the cable.

\section{DISCUSSION}

\section{A. The transition from 1:1 to $2: 1$ rhythm}

A transition from $1: 1$ to $2: 1$ rhythm is often seen in experimental $^{17,18,30,35,63-67,69-71}$ and modeling ${ }^{22,67,72}$ work on ventricular muscle and Purkinje fiber as the pacing frequency is increased or when some intervention is made that decreases the effective stimulus amplitude or the excitability of the tissue, e.g., ischemia, ${ }^{3,6}$ elevation of $\left[\mathrm{K}^{+}\right]_{o} .{ }^{73,74}$ This transition to $2: 1$ rhythm can be direct or indirect. For example, in paced isolated rabbit ventricular cells, the transition is direct when the stimulus amplitude is intermediate in size. ${ }^{67}$ In contrast, when the stimulus amplitude is higher or lower, alternans ${ }^{17}$ or Wenckebach ${ }^{63,71}$ rhythms are seen, respectively, before the $2: 1$ rhythm occurs. Similar results are found in aggregates of driven spontaneously beating embryonic chick ventricular cells. ${ }^{64}$ It remains to be seen in our model whether changing some parameter other than $\left[\mathrm{K}^{+}\right]_{o}$ (e.g., the length of the ischemic zone, the inter-cellular coupling within the ischemic zone, ${ }^{72}$ or the basic cycle length ${ }^{72}$ ), might result in a direct transition to $2: 1$ rhythm or an indirect transition via Wenckebach rhythms as $\left[\mathrm{K}^{+}\right]_{o}$ is then increased. It also remains to be seen whether a transition from 
$2: 2$ to $4: 4$ rhythm, and not to $2: 1$ rhythm, might occur, as has been reported in fast driving of toad ventricle ${ }^{75}$ and Purkinje fiber. ${ }^{35,68}$

In the cable simulations we report on above, the transition is indirect, since alternans is seen before 2:1 rhythm occurs (Figs. 3-5). In thin pieces of ventricular endocardium uniformly exposed to high $\left[\mathrm{K}^{+}\right]_{o}$, alternans is also seen before a 2:1 response occurs as $\left[\mathrm{K}^{+}\right]_{o}$ is elevated in the bathing solution. ${ }^{74}$ The alternans in that case involves two fullsized action potentials, as occurs within both normal zones in our case [e.g., Fig. 3(a): $x=0.75 \mathrm{~cm}$ and $x=2.25 \mathrm{~cm}$ ]. However, the alternans within the ischemic zone here involves the maintained small-amplitude response [e.g., Fig. 3(a): $x$ $=1.65 \mathrm{~cm}]$.

\section{B. Ischemic alternans}

Alternans is quite frequently seen in the electrocardiogram or electrogram in the acute stage of myocardial ischemia, in both clinical and experimental work (see the references given in the Introduction). There are also recordings of the transmembrane potential showing a beat-to-beat alternation in the action potential morphology during ischemia (e.g., Refs. 3, 6, 8). Indeed, the $\{1: 1 \rightarrow 2: 2 \rightarrow 2: 1\}$ progression we see above in the distal half of the cable as $\left[\mathrm{K}^{+}\right]_{o}$ is raised is exactly what is seen within the first few minutes of ischemia, with the reverse sequence being seen after the coronary occlusion is then released (see, e.g., Fig. 3 of Ref. 3).

The exact mechanisms underlying the ischemic alternans seen with any of the above three modes of recording remain unknown. Nevertheless, it is of interest to note that regional hyperkalemia induces alternans and ventricular arrhythmias. ${ }^{76}$ It is not known whether the alternation seen in the action potential morphology is "primary" (i.e., intrinsic to the cell) or whether it is "secondary" (i.e., due to electrotonic coupling with neighboring regions showing a 2:1 response). ${ }^{1,72}$ One can make the case for both of these forms of alternans in our model. Primary alternans is seen throughout the cable when there is a 2:2 rhythm [e.g., Fig. 3(a)]. In addition, a 2:2 rhythm closely resembling that seen within the ischemic zone in the cable simulations is seen in our simulations of space-clamped $\mathrm{K}^{+}$-depolarized membrane. However, the fact that the range of $\left[\mathrm{K}^{+}\right]_{o}$ over which the $2: 2$ rhythm exists in the cable is so narrow (only $\sim 0.05 \mathrm{mM}$ wide in Fig. 5) leads one to the conclusion that primary alternans might be quite rare during ischemia. Alternans is also seen in the cable during $2: 1$ block over a much wider range of $\left[\mathrm{K}^{+}\right]_{o}(\sim 0.2 \mathrm{mM})$; it then occurs throughout the entire proximal normal- $\left[\mathrm{K}^{+}\right]_{o}$ segment [e.g., Fig. 3(b): $x$ $=0.75 \mathrm{~cm}]$ and within the proximal part of the high- $\left[\mathrm{K}^{+}\right]_{o}$ ischemic central zone [Fig. 3(b): $x=1.35 \mathrm{~cm}$ ]. Alternans in the normal- $\left[\mathrm{K}^{+}\right]_{o}$ segment of tissue has also been seen in a Purkinje fiber strand where $\left[\mathrm{K}^{+}\right]_{o}$ is elevated along a central $8 \mathrm{~mm}$ long segment to produce a $2: 1$ block. $^{73}$

One interesting finding here is the small-amplitude deflection seen during 2:2 rhythm [e.g., at $x=1.65 \mathrm{~cm}$ in Fig. 3(a)]. When such a small deflection is recorded experimentally at one site, the assumption is usually made that this is a subthreshold response that is in the distal part of a pathway displaying decremental conduction. However, our simulations show that this is not necessarily the case, since this response can in fact be a nondecrementing response.

\section{Triggered alternans}

Our simulations show that alternans can be triggered by a pause in stimulation during 1:1 rhythm (Fig. 7) and detriggered by an extrastimulus. As pointed out earlier (Introduction), the sudden appearance or disappearance of alternans following a premature beat has been noted during experiments on ischemia several times. ${ }^{2,4,8,9}$ It is not completely clear from these studies whether it is the premature beat itself or the subsequent compensatory pause that is responsible for the flip from one rhythm to the other. The latter prospect is supported by the fact that a deliberate pause in stimulation can result in the triggering of alternans, ${ }^{8}$ which is reminiscent of the pause-induced triggering shown in Fig. 7. However, as already mentioned, the range of $\left[\mathrm{K}^{+}\right]_{o}$ over which the $2: 2$ rhythm is bistable with the $1: 1$ rhythm in our model is so narrow $(\sim 0.05 \mathrm{mM}$ in Fig. 5$)$, that one would not expect to stay within the bistable range of $\left[\mathrm{K}^{+}\right]_{o}$ for a very long time during rapidly evolving acute myocardial ischemia. It is of course possible that the range in the model is too narrow because of some deficiency in the model (e.g., the simple elevation of $\left[\mathrm{K}^{+}\right]_{o}$ might not be enough to accurately represent ischemia). ${ }^{28,38,39,42,59}$

In another experiment, ${ }^{4}$ the premature beat preceding triggering of alternans was quite late, so that the compensatory pause preceding triggering was not very long. Should the pause indeed be the cause of the triggering in that case, such a short pause would almost certainly not be long enough to produce paused-induced triggering of alternans in our model. Whereas detriggering of alternans can occur following a spontaneous premature beat and compensatory pause $^{2,4,8}$ or an intentional pause in stimulation, ${ }^{8}$ a pause during alternans does not detrigger the alternans in our model. This suggests that a different mechanism might be involved in detriggering than in triggering, or even that the mechanism of pause-induced triggering seen in our model might have little to do with the mechanism of pause-induced triggering during ischemia.

More than a century ago, Gaskell suggested that one way to account for mechanical alternans would be if "certain portions of the ventricle respond only to every second impulse, while other portions respond to every stimulus." 77 One intriguing possibility is thus that the triggering of alternans in the experiments might be due to the induction of a region of 2:1 block (which would then result in secondary alternans), since it is known that a pause can result in a flip from $1: 1$ to 2:1 rhythm in the intact ventricle ${ }^{69}$ and in isolated rabbit ventricular cells, ${ }^{67}$ as can an injection of a suitably timed extrastimulus in isolated ventricular cells. ${ }^{67}$ An intentionally inserted extrastimulus can also convert 2:1 into 1:1 rhythm, provided that the timing is right, ${ }^{67,69}$ suggesting a mechanism that might be capable of producing detriggering of secondary alternans. However, a pause in this circumstance would again not be expected to detrigger the secondary alternans, 
since a pause does not convert $2: 1$ to $1: 1$ rhythm in isolated rabbit ventricular cells. ${ }^{67}$ One can even speculate, should it be possible to flip back from alternans to $1: 1$ rhythm, that this maneuver might remove the well-known proarrhythmic effect of alternans. Further systematic experimental work exploring the origin of triggering and detriggering of alternans during ischemia is thus indicated.

A flip between high- and low-amplitude ischemic alternans following an extrasystole has been described. ${ }^{5,6,8}$ There is also evidence for this bistability (indeed, perhaps multistability or neutral stability) during mechanical alternans of the nonischemic ventricle. ${ }^{78-80}$ Thus, by our definition of triggered alternans, it is important to rule out the possibility that there is a pre-existing very-low-amplitude alternans present [see, e.g., Fig. 3 of Ref. 2; Fig. 5(a) of Ref. 8] before stating that triggered alternans occurs. There is evidence that the flip from a lower-amplitude to a higher-amplitude alternans during ischemia is due to the conversion of discordant alternans (alternation $180^{\circ}$ out-of-phase at some sites) into concordant alternans (alternation in-phase at all sites). ${ }^{5,6}$ While there have been modeling studies showing discordant alternans in nonischemic models, ${ }^{29-31}$ it remains to be seen whether discordant alternans will be seen in models of ischemic muscle, and whether flips between the concordant and discordant forms of alternans will be possible. Such flips can be seen in a homogeneous cable model ${ }^{29}$ and in an elegantly simple phenomenological model. ${ }^{19}$

\section{Bistability involving $1: 1,2: 2$, and $2: 1$ rhythms}

Four forms of bistability involving $1: 1,2: 2$, and $2: 1$ rhythms have been described: $\{2: 2 \leftrightarrow 2: 1\},\{1: 1 \leftrightarrow 2: 1\}$, $\{1: 1 \leftrightarrow 2: 2\}$, and $\left\{2: 2_{A} \leftrightarrow 2: 2_{B}\right\}$ (i.e., bistability between two different $2: 2$ rhythms).

First, pulse- or pause-induced flips can be seen between $2: 2$ and $2: 1$ rhythms in isolated rabbit ventricular cells, ${ }^{17}$ hysteresis between these two rhythms (suggesting the existence of bistability) has been described in bullfrog ventricle, ${ }^{66}$ and either a $2: 2$ or a $2: 1$ rhythm can be seen in a homogeneous 1-dimensional Beeler-Reuter cable, depending on initial conditions. ${ }^{27}$ The existence of the $\{2: 2 \leftrightarrow 2: 1\}$ bistability is predicted by a simple one-dimensional twobranched discontinuous map derived from the APD restitution curve, and involves the co-existence of a stable period-1 orbit on one branch of the map with a stable period-doubled period-2 orbit on the other branch of the map. ${ }^{27,33}$

Second, $\{1: 1 \leftrightarrow 2: 1\}$ bistability is found in frog ventricle, ${ }^{69}$ in aggregates of embryonic chick ventricular cells, ${ }^{64}$ in single rabbit ventricular cells, ${ }^{67}$ in the human HisPurkinje system, ${ }^{81}$ and in the space-clamped Luo-Rudy model. ${ }^{67}$ Hysteresis between these two rhythms has also been described. ${ }^{24,64,66,67,69}$ The $\{1: 1 \leftrightarrow 2: 1\}$ bistability is predicted by a simple one-dimensional two-branched map derived from the APD restitution curve, ${ }^{27,33,67}$ and involves the co-existence of two different period-1 points on the two branches of the discontinuous map ${ }^{27,33,67}$ (but see Ref. 66 for a dissenting view).

Third, we show an example of $\{1: 1 \leftrightarrow 2: 2\}$ bistability in Fig. 7. Apart from the triggered ischemic alternans men- tioned above, the only other two instances of $\{1: 1 \leftrightarrow 2: 2\}$ bistability of which we are aware occur in the mechanical contraction of the heart ${ }^{78-80}$ and in periodic driving of modified forms of the space-clamped Beeler-Reuter equations. $^{22-24}$ In the latter case, the behavior is different from ours, in that the $2: 2$ cycle is made up of two full-sized action potentials [compare, e.g., the inset in Fig. 4(b) of Ref. 23 with our Fig. 3(a)]. An iterative analysis ${ }^{32}$ was carried out for that case using the APD restitution curve. ${ }^{22}$ This analysis can be transformed into consideration of a one-dimensional $\left(\mathrm{APD}_{i}, \mathrm{APD}_{i+1}\right)$ map having a single branch of negative slope, ${ }^{27,33}$ which is in contrast to our effectively twobranched map [Fig. 8(c)]. In a differently modified version of the Beeler-Reuter model, the iterative approach predicted that there would be bistability between $1: 1$ and $2: 2$ rhythms, ${ }^{23,24,26}$ due to the coexistence of stable period- 1 and period-2 orbits on the single branch of the map (when the Schwarzian derivative is negative for a unimodal map, there can be at most one stable periodic orbit). ${ }^{82}$ An unstable period- 2 orbit then serves to separate the basins of attraction of the stable period- 1 and the stable period- 2 orbits, which is not the case in Fig. 6(c), where the discontinuity and its pre-image serve that purpose. In the ionic modeling work, the unstable period-2 orbit arises from a subcritical perioddoubling bifurcation that occurs as the bifurcation parameter (the basic cycle length) is decreased. ${ }^{23,26}$ Very subtle changes in the APD restitution curve can result in the subcritical bifurcation becoming supercritical, thus destroying the unstable period-2 orbit and the bistability. ${ }^{26}$ As in our case (Fig. 5 ), the bistability found in the numerical simulations existed only over an extremely narrow range of the bifurcation parameter (as little as $1 \mathrm{~ms}$ of basic cycle length). ${ }^{23,24}$ This bistability in the space-clamped system has been linked with the bistability between periodic and quasiperiodic modes of sustained reentry around a one-dimensional loop. ${ }^{24}$ We know of no experimental report in single cells of this kind of bistability.

Fourth, bistability has been described between two different 2:2 rhythms in frog ventricle, ${ }^{32}$ and a bistability or multistability or neutral stability between 2:2 rhythms has been described in the mechanical activity of the dog heart. ${ }^{78-80}$ This bistability has also been treated in terms of a single-branched one-dimensional map. ${ }^{27}$ In addition, as detailed above, concordant and discordant alternans can be viewed as coexisting 2:2 rhythms.

\section{E. Nonischemic alternans; alternans control}

While alternans can be a precursor of ventricular arrhythmias in situations not involving ischemia (e.g., rapid pacing, ${ }^{83}$ long-QT syndrome, ${ }^{15}$ administration of antiarrhythmic drugs ${ }^{84}$ ), we are not aware of any reports of triggered alternans in these nonischemic cases. Our expectation is that triggered alternans will show up in these and other situations in which alternans is know to occur, once a systematic search is made.

Alternans in the atrioventricular conduction time, induced in human beings by a pacing protocol, can be made to revert to a 1:1 atrioventricular rhythm by using a modifica- 
tion of a "chaos-control" technique, which involves reduction of the dynamics to consideration of a one-dimensional map. ${ }^{85}$ The excellent approximation of the dynamics described above by a one-dimensional map makes it more feasible that ischemic alternans, a well-known precursor of malignant arrhythmias, can be made to revert back to a less ominous period- 1 rhythm by means of some sort of "alternans control" technique.

\section{ACKNOWLEDGMENTS}

We thank CONACYT Grant No. 32164-E (H.A., A.L.), PAPIME-UNAM Grant No. 191054 (H.A.), and the Canadian Institutes of Health Research (M.R.G.) for financial support, and Enrique Palacios-Boneta for general help with computers.

${ }^{1}$ H. Arce, A. Xu, H. González, and M. R. Guevara, “Alternans and higherorder rhythms in an ionic model of a sheet of ischemic ventricular muscle," Chaos 10, 411-426 (2000).

${ }^{2}$ H. K. Hellerstein and I. M. Liebow, "Electrical alternation in experimental coronary artery occlusion," Am. J. Physiol. 160, 366-374 (1950).

${ }^{3}$ E. Downar, M. J. Janse, and D. Durrer, "The effect of acute coronary artery occlusion on subepicardial transmembrane potentials in the intact porcine heart," Circulation 56, 217-224 (1977).

${ }^{4}$ K. Kataoka and R. Yoshimura, "Clinical and experimental studies on electrical alternans of ST-segment and T-wave in variant form of angina pectoris (in Japanese)," Resp. Circ. 27, 767-776 (1979).

${ }^{5}$ H. Hashimoto, K. Suzuki, and M. Nakashima, "Effects of the ventricular premature beat on the alternation of the repolarization phase in ischemic myocardium during acute coronary occlusion in dogs," J. Electrocardiol. 17, 229-238 (1984)

${ }^{6} \mathrm{H}$. Hashimoto, M. Asano, and M. Nakashima, "Potentiating effects of a ventricular premature beat on the alternation of the ST-T complex of epicardial electrograms and the incidence of ventricular arrhythmias during acute coronary occlusion in dogs," J. Electrocardiol. 17, 289-301 (1984).

${ }^{7}$ D. L. Carson, R. Cardinal, P. Savard, and M. Vermeulen, "Characterisation of unipolar waveform alternation in acutely ischaemic porcine myocardium," Cardiovasc. Res. 20, 521-527 (1986).

${ }^{8}$ S. G. Dilly and M. J. Lab, "Electrophysiological alternans and restitution during acute regional ischaemia in myocardium of anaesthetized pig," J. Physiol. (London) 402, 315-333 (1988).

${ }^{9}$ R. Mohabir, M. R. Franz, and W. T. Clusin, "In vivo electrophysiological detection of myocardial ischemia through monophasic action potential recording," Prog. Cardiovasc. Dis. 34, 15-28 (1991).

${ }^{10}$ D. R. Adam, S. Akselrod, and R. J. Cohen, "Estimation of ventricular vulnerability to fibrillation through T-wave time series analysis," Computers in Cardiology (IEEE, New York, 1982), pp. 307-310.

${ }^{11}$ D. R. Adam, J. M. Smith, S. Akselrod, S. Nyberg, A. O. Powell, and R. J. Cohen, "Fluctuations in T-wave morphology and susceptibility to ventricular fibrillation," J. Electrocardiol. 17, 209-218 (1984).

${ }^{12}$ S. Horinaka, S. Hara, N. Tsuchiya, A. Yabe, H. Asakawa, H. Yagi, Y. Mori, and H. Matsuoka, "Alternans of ventricular gradient during percutaneous transluminal coronary angioplasty," J. Electrocardiol. 34, 135-139 (2001).

${ }^{13}$ B. D. Nearing, A. H. Huang, and R. L. Verrier, "Dynamic tracking of cardiac vulnerability by complex demodulation of the T wave," Science 252, 437-440 (1991).

${ }^{14}$ P. Laguna, G. B. Moody, J. Garcia, A. L. Goldberger, and R. G. Mark, "Analysis of the ST-T complex of the electrocardiogram using the Karhunen-Loeve transform: adaptive monitoring and alternans detection," Med. Biol. Eng. Comput. 37, 175-189 (1999).

${ }^{15}$ S. B. Platt, J. M. Vijgen, P. Albrecht, G. F. van Hare, M. D. Carlson, and D. S. Rosenbaum, "Occult T wave alternans in long QT syndrome," J. Cardiovasc. Electrophysiol. 7, 144-148 (1996).

${ }^{16} \mathrm{~S}$. H. Hohnloser, T. Klingenheben, Y.-G. Li, M. Zabel, J. Peetermans, and R. J. Cohen, "T wave alternans as a predictor of recurrent ventricular tachyarrhythmias in ICD recipients: prospective comparison with conventional risk markers," J. Cardiovasc. Electrophysiol. 9, 1258-1268 (1998).

${ }^{17}$ M. R. Guevara, F. Alonso, D. Jeandupeux, and A. C. G. van Ginneken,
"Alternans in periodically stimulated isolated ventricular myocytes: Experiment and model," in Cell to Cell Signalling: From Experiments to Theoretical Models, edited by A. Goldbeter (Harcourt Brace Jovanovich, London, 1989), pp. 551-563.

${ }^{18}$ J. Hescheler and R. Speicher, "Regular and chaotic behaviour of cardiac cells stimulated at frequencies between 2 and $20 \mathrm{~Hz}$," Eur. Biophys. J. 17, 273-280 (1989).

${ }^{19}$ D. S. Rubenstein and S. L. Lipsius, "Premature beats elicit a phase reversal of mechanoelectrical alternans in cat ventricular myocytes: A possible mechanism for reentrant arrhythmias," Circulation 91, 201-214 (1995).

${ }^{20}$ E. Chudin, J. Goldhaber, A. Garfinkel, J. Weiss, and B. Kogan, "Intracellular $\mathrm{Ca}^{2+}$ dynamics and the stability of ventricular tachycardia," Biophys. J. 77, 2930-2941 (1999).

${ }^{21}$ J. Hüser, Y. G. Wang, K. A. Sheehan, F. Cifuentes, S. L. Lipsius, and L. A. Blatter, "Functional coupling between glycolysis and activationcontraction coupling underlies alternans in cat heart cells," J. Physiol. (London) 524, 795-806 (2000).

${ }^{22}$ A. Vinet, D. R. Chialvo, D. C. Michaels, and J. Jalife, "Nonlinear dynamics of rate-dependent activation in models of single cardiac cells," Circ. Res. 67, 1510-1524 (1990).

${ }^{23}$ A. Vinet and F. A. Roberge, "Analysis of an iterative difference equation model of the cardiac cell membrane," J. Theor. Biol. 170, 201-214 (1994).

${ }^{24}$ A. Vinet, "Memory and bistability in a one-dimensional loop of model cardiac cells," J. Biol. Syst. 7, 451-473 (1999).

${ }^{25}$ J. J. Fox, J. L. McHarg, and R. F. Gilmour, Jr., "Ionic mechanism of electrical alternans," Am. J. Physiol. 282, H516-H530 (2001).

${ }^{26}$ A. Vinet, "Quasiperiodic circus movement in a loop model of cardiac tissue: multistability and low dimensional equivalence," Ann. Biomed. Eng. 28, 704-720 (2000).

${ }^{27}$ T. J. Lewis and M. R. Guevara, "Chaotic dynamics in an ionic model of the propagated cardiac action potential," J. Theor. Biol. 146, 407-432 (1990).

${ }^{28}$ J. M. Ferrero, Jr., B. Rodriguez, B. Trenor et al., "Action potential duration inhomogeneities in acute myocardial ischemia: a simulation study," Computers in Cardiology (IEEE, New York, 1998), pp. 577-580.

${ }^{29}$ M. A. Watanabe, F. H. Fenton, S. J. Evans, H. M. Hastings, and A. Karma, "Mechanisms for discordant alternans," J. Cardiovasc. Electrophysiol. 12, 196-206 (2001)

${ }^{30}$ J. J. Fox, M. L. Riccio, F. Hua, E. Bodenschatz, and R. F. Gilmour, Jr., "Spatiotemporal transition to conduction block in canine ventricle," Circ. Res. 90, 289-296 (2002).

${ }^{31}$ Z. Qu, A. Garfinkel, P.-S. Chen, and J. N. Weiss, "Mechanisms of discordant alternans and induction of reentry in simulated cardiac tissue," Circulation 102, 1664-1670 (2000).

${ }^{32}$ J. B. Nolasco and R. W. Dahlen, "A graphic method for the study of alternation in cardiac action potentials," J. Appl. Physiol. 25, 191-196 (1968)

${ }^{33}$ M. R. Guevara, G. Ward, A. Shrier, and L. Glass, "Electrical alternans and period-doubling bifurcations," Computers in Cardiology (IEEE Computer Society, Silver Springs, MD, 1984), pp. 167-170.

${ }^{34}$ M. R. Guevara and A. Shrier, "Rhythms produced by high-amplitude periodic stimulation of spontaneously beating aggregates of embryonic chick ventricular myocytes," Ann. N.Y. Acad. Sci. 591, 11-22 (1990).

${ }^{35}$ D. R. Chialvo, R. F. Gilmour, Jr., and J. Jalife, "Low dimensional chaos in cardiac tissue," Nature (London) 343, 653-657 (1990).

${ }^{36}$ J.-M. Cao, Z. Qu, Y.-H. Kim, T.-J. Wu, A. Garfinkel, J. N. Weiss, H. S. Karagueuzian, and P.-S. Chen, "Spatiotemporal heterogeneity in the induction of ventricular fibrillation by rapid pacing. Importance of cardiac restitution properties," Circ. Res. 84, 1318-1331 (1999).

${ }^{37} \mathrm{~B}$. Tilg and P. Wach, "Simulation of the 1-D cardiac excitation process by varying extracellular potassium concentration and intracellular resistivity," Computers in Cardiology (IEEE, New York, 1995), pp. 421-424.

${ }^{38}$ J. M. Ferrero, Jr., V. Torres, J. Saiz, M. Monserrat, J. M. Ferrero, and N. V. Thakor, "Simulation study of action potentials during acute myocardial ischemia," Comput. Cardiol. 24, 705-708 (1997).

${ }^{39}$ R. M. Shaw and Y. Rudy, "Electrophysiologic effects of acute myocardial ischemia. A mechanistic investigation of action potential conduction and conduction failure," Circ. Res. 80, 124-138 (1997).

${ }^{40}$ A. Cimponeriu, C. F. Starmer, and A. Bezerianos, "Action potential propagation in ischemic cardiac tissue: a theoretical computer modeling," in Ref. 28, pp. 317-320.

${ }^{41} \mathrm{~A}$. Xu and M. R. Guevara, "Two forms of spiral-wave reentry in an ionic model of ischemic ventricular myocardium," Chaos 8, 157-174 (1998). 
${ }^{42}$ A. B. Feldman, H. D. Esperer, and R. J. Cohen, "A mechanism controlling the characteristics of T-wave alternans measurements in the setting of myocardial ischemia," Comput. Cardiol. 26, 149-152 (1999).

${ }^{43}$ Y. Wang and Y. Rudy, "Action potential propagation in inhomogeneous cardiac tissue: safety factor considerations and ionic mechanism," Am. J. Physiol. 278, H1019-H1029 (2000).

${ }^{44}$ C.-h. Luo and Y. Rudy, "A model of the ventricular cardiac action potential. Depolarization, repolarization, and their interaction," Circ. Res. 68, 1501-1526 (1991)

${ }^{45}$ S. Guan, Q. Lu, and K. Huang, "A discussion about the DiFrancescoNoble model," J. Theor. Biol. 189, 27-32 (1997).

${ }^{46}$ A. Varghese and G. R. Sell, "A conservation principle and its effect on the formulation of $\mathrm{Na}-\mathrm{Ca}$ exchanger current in cardiac cells," J. Theor. Biol. 189, 33-40 (1997).

${ }^{47}$ L. P. Endresen, K. Hall, J. S. Høye, and J. Myrheim, "A theory for the membrane potential of living cells," Eur. Biophys. J. 29, 90-103 (2000).

${ }^{48}$ L. P. Endresen and N. Skarland, "Limit cycle oscillations in pacemaker cells," IEEE Trans. Biomed. Eng. 47, 1134-1137 (2000).

${ }^{49}$ S. Dokos, B. G. Celler, and N. H. Lovell, "Modification of DiFrancescoNoble equations to simulate the effects of vagal stimulation on in vivo mammalian sinoatrial node electrical activity," Ann. Biomed. Eng. 21, 321-335 (1993)

${ }^{50} \mathrm{~A}$. Varghese and R. L. Winslow, "Dynamics of the calcium subsystem in cardiac Purkinje fibers," Physica D 68, 364-386 (1993).

${ }^{51}$ A. Varghese and R. L. Winslow, "Dynamics of abnormal pacemaking activity in cardiac Purkinje fibers," J. Theor. Biol. 168, 407-420 (1994).

${ }^{52}$ T. J. Hund, J. P. Kucera, N. F. Otani, and Y. Rudy, "Ionic charge conservation and long-term steady state in the Luo-Rudy dynamic cell model," Biophys. J. 81, 3324-3331 (2001).

${ }^{53} \mathrm{G}$. W. Beeler and H. Reuter, "Reconstruction of the action potential of ventricular myocardial fibres," J. Physiol. (London) 268, 177-210 (1977).

${ }^{54} \mathrm{G}$. Isenberg and U. Klöckner, "Calcium currents of isolated bovine ventricular myocytes are fast and of large amplitude," Pfluegers Arch. 395, 30-41 (1982).

${ }^{55}$ G. H. Sharp and R. W. Joyner, "Simulated propagation of cardiac action potentials,” Biophys. J. 31, 403-423 (1980).

${ }^{56}$ L. Boucher and J.-P. Drouhard, "Analyse par simulation des effets de la discrétisation sur le potential d'action ventriculaire propagé." Institut de Génie Biomédical, Université de Montréal, Montréal. Technical Report, 1983.

${ }^{57}$ B. Victorri, F. A. Roberge, L. Boucher, A. Vinet, and J. P. Drouhard, in Ref. 33, pp. 431-434

${ }^{58} \mathrm{~J}$. Wu and D. P. Zipes, "Effects of spatial segmentation in the continuous model of excitation propagation in cardiac muscle," J. Cardiovasc. Electrophysiol. 10, 965-972 (1999).

${ }^{59}$ R. M. Shaw and Y. Rudy, "Electrophysiologic effects of acute myocardial ischemia: a theoretical study of altered cell excitability and action potential duration," Cardiovasc. Res. 35, 256-272 (1997).

${ }^{60}$ W. Mobitz, “Über die unvollständige Storung der Erregungsüberleitung zwischen Vorhof und Kammer des menschlichen Herzens," Z. Gesamte Exp. Med. 41, 180-237 (1924).

${ }^{61}$ A. Shrier, H. Dubarsky, M. Rosengarten, M. R. Guevara, S. Nattel, and L. Glass, "Prediction of complex atrioventricular conduction rhythms in humans with use of the atrioventricular nodal recovery curve," Circulation 76, 1196-1205 (1987).

${ }^{62}$ M. R. Guevara, L. Glass, and A. Shrier, "Phase locking, period-doubling bifurcations, and irregular dynamics in periodically stimulated cardiac cells," Science 214, 1350-1353 (1981).

${ }^{63}$ M. R. Guevara, D. Jeandupeux, F. Alonso, and N. Morissette, "Wenckebach rhythms in isolated ventricular heart cells," in International Conference on Singular Behavior and Nonlinear Dynamics, Vol. 2, edited by St. Pnevmatikos, T. Bountis, and Sp. Pnevmatikos (World Scientific, Singapore, 1989), pp. 629-642.

${ }^{64}$ M. R. Guevara, A. Shrier, and L. Glass, "Chaotic and complex cardiac rhythms," in Cardiac Electrophysiology: From Cell to Bedside, 1st ed., edited by D. P. Zipes and J. Jalife (Saunders, Philadelphia, 1990), pp. 192-201.
${ }^{65}$ M. L. Koller, M. L. Riccio, and R. F. Gilmour, Jr., "Dynamic restitution of action potential duration during electrical alternans and ventricular fibrillation,” Am. J. Physiol. 275, H1635-H1642 (1998).

${ }^{66}$ G. M. Hall, S. Bahar, and D. J. Gauthier, "Prevalence of rate-dependent behaviors in cardiac muscle," Phys. Rev. Lett. 82, 2995-2998 (1999).

${ }^{67}$ A. R. Yehia, D. Jeandupeux, F. Alonso, and M. R. Guevara, "Hysteresis and bistability in the direct transition from $1: 1$ to $2: 1$ rhythm in periodically driven single ventricular cells," Chaos 9, 916-931 (1999).

${ }^{68}$ R. F. Gilmour, Jr., N. F. Otani, and M. A. Watanabe, "Memory and complex dynamics in cardiac Purkinje fibers," Am. J. Physiol. 272, H1826H1832 (1997).

${ }^{69}$ G. R. Mines, "On dynamic equilibrium in the heart," J. Physiol. (London) 46, 349-383 (1913).

${ }^{70}$ D. R. Chialvo and J. Jalife, "Non-linear dynamics of cardiac excitation and impulse propagation," Nature (London) 330, 749-752 (1987).

${ }^{71}$ A. R. Yehia, A. Shrier, K. C.-L. Lo, and M. R. Guevara, "Transient outward current contributes to Wenckebach-like rhythms in isolated rabbit ventricular cells," Am. J. Physiol. 273, H1-H11 (1997).

${ }^{72}$ M. R. Guevara, "Spatiotemporal patterns of block in an ionic model of cardiac Purkinje fibre," in From Chemical to Biological Organization, edited by M. Markus, S. C. Müller, and G. Nicolis (Springer-Verlag, Berlin, 1988), pp. 273-281.

${ }^{73}$ P. F. Cranefield, H. O. Klein, and B. F. Hoffman, "Conduction of the cardiac impulse. 1. Delay, block, and one-way block in depressed Purkinje fibers," Circ. Res. 28, 199-219 (1971).

${ }^{74}$ M. L. Koller, M. L. Riccio, and R. F. Gilmour, Jr., "Effects of $\left[\mathrm{K}^{+}\right]_{o}$ on electrical restitution and activation dynamics during ventricular fibrillation," Am. J. Physiol. 279, H2665-H2672 (2000).

${ }^{75}$ G. V. Savino, L. Romanelli, D. L. González, O. Piro, and M. E. Valentinuzzi, "Evidence for chaotic behavior in driven ventricles," Biophys. J. 56, 273-280 (1989).

${ }^{76}$ M. J. Curtis, "The rabbit dual coronary perfusion model: a new method for assessing the pathological relevance of individual products of the ischaemic milieu: role of potassium in arrhythmogenesis," Cardiovasc. Res. 25, 1010-1022 (1991).

${ }^{77}$ W. H. Gaskell, "On the rhythm of the heart of the frog, and on the nature of the action of the vagus nerve," Philos. Trans. R. Soc. London, Ser. A 173, 993-1033 (1882).

${ }^{78}$ W. G. Guntheroth, B. C. Morgan, G. A. McGough, and A. M. Scher, "Alternate deletion and potentiation as the cause of pulsus alternans," Am. Heart J. 78, 669-681 (1969).

${ }^{79}$ Y. Mahler and S. Rogel, "Interrelation between restitution time-constant and alternating myocardial contractility in dogs," Clin. Sci. 39, 625-639 (1970).

${ }^{80}$ S. Suzuki, J. Araki, Y. Doi, W. Fujinaka, H. Minami, G. Iribe, S. Mohri, J. Shimizu, M. Hirakawa, and H. Suga, "Coupling interval from slow to tachycardic pacing decides sustained alternans pattern," Am. J. Physiol. 280, H1368-H1375 (2001)

${ }^{81}$ E. Pruvot, A. De Torrente, G. M. De Ferrari, P. J. Schwartz, and J.-J. Goy, "Two-to-one AV block associated with the congenital long QT syndrome," J. Cardiovasc. Electrophysiol. 10, 108-113 (1999).

${ }^{82}$ G. Mayer-Kress and H. Haken, "Transition to chaos for maps with positive Schwarzian derivative," in Evolution of Order and Chaos, edited by H. Haken (Springer-Verlag, Berlin, 1982), pp. 183-186.

${ }^{83}$ J. M. Pastore, S. D. Girouard, K. R. Laurita, F. G. Akar, and D. S. Rosenbaum, "Mechanism linking T-wave alternans to the genesis of cardiac fibrillation," Circulation 99, 1385-1394 (1999).

${ }^{84}$ H. Tachibana, M. Yamaki, I. Kubota, T. Watanabe, S. Yamauchi, and H. Tomoike, "Intracoronary flecainide induces ST alternans and reentrant arrhythmia on intact canine heart. A role of 4-aminopyridine-sensitive current," Circulation 99, 1637-1643 (1999).

${ }^{85}$ D. J. Christini, K. M. Stein, S. M. Markowitz, S. Mittal, D. J. Slotwiner, M. A. Scheiner, S. Iwai, and B. B. Lerman, "Nonlinear-dynamical arrhythmia control in humans," Proc. Natl. Acad. Sci. U.S.A. 98, 58275832 (2001) 
Chaos is copyrighted by the American Institute of Physics (AIP). Redistribution of journal material is subject to the AIP online journal license and/or AIP copyright. For more information, see http://ojps.aip.org/chaos/chocr.jsp

Copyright of Chaos is the property of American Institute of Physics and its content may not be copied or emailed to multiple sites or posted to a listserv without the copyright holder's express written permission. However, users may print, download, or email articles for individual use. 